\title{
Collider signatures of a light NMSSM pseudoscalar in neutralino decays in the light of LHC results
}

\author{
David G. Cerdeño, ${ }^{a, b}$ Pradipta Ghosh, ${ }^{a, b}$ Chan Beom Park $^{c}$ and Miguel Peiró ${ }^{a, b}$ \\ a Departamento de Física Teórica, Universidad Autónoma de Madrid, \\ Cantoblanco, E-28049 Madrid, Spain \\ ${ }^{b}$ Instituto de Física Teórica UAM/CSIC, Universidad Autónoma de Madrid, \\ Cantoblanco, E-28049 Madrid, Spain \\ ${ }^{c}$ TH Division, Physics Department, CERN, \\ CH-1211 Geneva 23, Switzerland \\ E-mail: davidg.cerdeno@uam.es, pradipta.ghosh@uam.es, \\ chanbeom.park@cern.ch, miguel.peiro@uam.es
}

ABSTRACT: We investigate signatures induced by a very light pseudoscalar Higgs in neutralino decays in the Next-to-Minimal Supersymmetric Standard Model (NMSSM) and determine their observability at the LHC. We concentrate on scenarios which feature two light scalar Higgs bosons (one of them is SM-like with a mass of $125 \mathrm{GeV}$ and a singlet-like lighter one) with a very light (singlet-like) pseudoscalar Higgs in the mass range $2 m_{\tau}<m_{a_{1}^{0}}<2 m_{b}$. We consider neutralino-chargino pair production and the subsequent decay $\widetilde{\chi}_{2,3}^{0} \rightarrow \widetilde{\chi}_{1}^{0} a_{1}^{0}$, which leads to topologies involving multi-leptons and missing transverse energy. We determine a set of selection cuts that can effectively isolate the signal from backgrounds of the Standard Model or the Minimal Supersymmetric Standard Model. We also exemplify the procedure with a set of benchmark points, for which we compute the expected number of events and signal strength for LHC with $8 \mathrm{TeV}$ center of mass energy. We show that this signal can already be probed for some points in the NMSSM parameter space.

KeYwords: Supersymmetry Phenomenology

ARXIV EPRINT: 1307.7601 


\section{Contents}

1 Introduction 1

2 Light Higgs scenarios in the NMSSM and choice of benchmark points 2

3 Direct production of neutralinos decaying into a light pseudoscalar $\quad 10$

$\begin{array}{lll}4 & \text { Conclusions } & 17\end{array}$

\section{Introduction}

The Next-to-Minimal Supersymmetric Standard Model (NMSSM) (see, e.g., ref. [1] for a review) is a well motivated extension of the MSSM. In the NMSSM a new singlet superfield $\hat{S}$ is included in order to provide a dynamical mechanism by which the Higgsino mass parameter, $\mu$, is naturally of the order of the electroweak (EW) scale, thereby addressing the so-called " $\mu$ problem" [2]. The NMSSM leads to a very interesting Higgs phenomenology, due to the presence of an extra scalar Higgs and a pseudoscalar Higgs. These states can be very light without violating current collider constraints, provided that they are mostly singlet-like. Moreover, within the NMSSM a new contribution to the tree level Higgs mass [3-7], coming from the $\lambda \hat{S} \hat{H}_{u} \hat{H}_{d}$ term in the superpotential, makes it easier to obtain a relatively heavy Standard Model (SM) like Higgs boson while reducing somehow the fine-tuning [8-18]. This is favoured by the LHC observation of a Higgs boson with a mass in the $2 \sigma$ range $124-126.8 \mathrm{GeV}(124.5-126.9 \mathrm{GeV})$ by ATLAS (CMS) [19-22]. It has also been shown that in some regions of the parameter space another light, singlet-like, Higgs $\left(h_{1}^{0}\right)$ can also be present, with a mass around $98 \mathrm{GeV}$ [23-27], motivated by the small excess in the LEP search for $e^{+} e^{-} \rightarrow Z h, h \rightarrow b \bar{b}$ [28-30], or even lighter [26, 31-35].

Some of these scenarios also present a very light pseudoscalar Higgs boson, $a_{1}^{0}$, [26, 27, 31-34], which is very appealing from the point of view of LHC signatures. For example, in ref. [26] we investigated how a very light pseudoscalar in the mass range $2 m_{\tau}<m_{a_{1}^{0}}<2 m_{b}$ could be probed in multilepton decays of the scalar Higgses, $h_{1,2}^{0} \rightarrow a_{1}^{0} a_{1}^{0} \rightarrow 4 \ell+\mathbb{E}_{\mathrm{T}}$ (where $\ell=\mathrm{e}, \mu$, and hadronically-decaying $\tau, \tau_{h}$ and $\mathbb{E}_{\mathrm{T}}$ denotes the missing transverse energy), and showed how the pseudoscalar mass can be reconstructed. Another remarkable effect of a light pseudoscalar is that it can be copiously produced in the decays of neutralinos $\widetilde{\chi}_{2,3}^{0} \rightarrow \widetilde{\chi}_{1}^{0} a_{1}^{0}$, constituting a characteristic NMSSM signature [35-39]. It should be noted that these scenarios are extremely sensitive to the Higgs properties and therefore very affected by the recent experimental constraints. For example, the latest measurements of the SM-like Higgs $\left(h_{\mathrm{SM}}\right)[21,22]$ forbid a sizable contribution to non-SM decay modes, such as $h_{\mathrm{SM}} \rightarrow a_{1}^{0} a_{1}^{0}, h_{\mathrm{SM}} \rightarrow h_{1}^{0} h_{1}^{0} / h_{1}^{0^{*}}$ or $h_{\mathrm{SM}} \rightarrow \widetilde{\chi}_{1}^{0} \widetilde{\chi}_{1}^{0}$, and therefore motivates the reanalysis of the phenomenology associated to neutralino decays. 
In this work we concentrate on scenarios which feature two light scalar Higgses (one of them SM-like with a mass of approximately $125 \mathrm{GeV}$ and a singlet-like lighter one) with a very light singlet-like pseudoscalar (in the mass range $2 m_{\tau}<m_{a_{1}^{0}}<2 m_{b}$ ). We carry out a systematic search for regions of the NMSSM parameter space in which the branching ratio $\operatorname{BR}\left(\widetilde{\chi}_{2,3}^{0} \rightarrow \widetilde{\chi}_{1}^{0} a_{1}^{0}\right)$ is sizable. For the range of masses considered, the pseudoscalar predominantly decays into a pair of taus, $a_{1}^{0} \rightarrow \tau^{+} \tau^{-}$, leading to an abundance of leptons in the final state. Therefore, the resulting LHC phenomenology features multilepton signals with missing transverse energy in the decay chains after neutralino/chargino pair production, $\widetilde{\chi}_{2,3}^{0} \widetilde{\chi}_{1}^{ \pm} \rightarrow \ell^{+} \ell^{-} \ell^{ \pm}+\mathbb{E}_{\mathrm{T}}, \widetilde{\chi}_{3}^{0} \widetilde{\chi}_{1}^{ \pm} \rightarrow 2 \ell^{+} 2 \ell^{-} \ell^{ \pm}+\mathbb{E}_{\mathrm{T}}$, and $\widetilde{\chi}_{i}^{0} \widetilde{\chi}_{j}^{0} \rightarrow n\left(\ell^{+} \ell^{-}\right)+\mathbb{E}_{\mathrm{T}}$, with $n=2,3,4$ and $i, j=2,3$.

Multi-lepton final states have always been considered an important probe for supersymmetry (SUSY) searches in colliders [40-70]. The unusual source of leptons $\left(a_{1}^{0} \rightarrow \tau^{+} \tau^{-}\right)$ considered in this work within the context of the NMSSM with a pair of light scalar Higgses requires the modification of conventional search strategies. In this paper, we define a set of event selection cuts that will allow the studied signal to be distinguished from the SM and MSSM backgrounds. All the recent experimental bounds on the Higgs sector are included, as well as the constraints on the masses of supersymmetric particles and low-energy observables. We also take into account bounds on the neutralino relic abundance and on its elastic scattering cross-section off quarks.

The paper is organised as follows. In section 2, we present the results of a scan in the NMSSM parameter space, applying the most recent experimental constraints on Higgs sector and low-energy observables as well as constraints from dark matter searches. We also determine the regions of interest for our analysis. In section 3, we describe the collider phenomenology of the signal and define a set of effective event selection cuts for background suppression. We further estimate the relevant backgrounds and the signal significance of the resulting signatures for some selected benchmark points. Finally, we present our concluding remarks in section 4 .

\section{Light Higgs scenarios in the NMSSM and choice of benchmark points}

The $\mathbb{Z}_{3}$ invariant NMSSM superpotential (see e.g., [1]) reads

$$
W=W_{\mathrm{MSSM}}^{\prime}-\epsilon_{a b} \lambda \hat{S} \hat{H}_{d}^{a} \hat{H}_{u}^{b}+\frac{1}{3} \kappa \hat{S}^{3},
$$

where $W_{\text {MSSM }}^{\prime}$ is the MSSM superpotential [71] without the bilinear $\epsilon_{a b} \mu \hat{H}_{d}^{a} \hat{H}_{u}^{b}$ term, with $\epsilon_{12}=1 . \hat{H}_{u}, \hat{H}_{d}$ are two $\mathrm{SU}(2)$-doublet Higgs superfields and $\hat{S}$ is a new superfield, singlet under the SM gauge group. The superpotential incorporates two new couplings, $\lambda$ and $\kappa$. The Lagrangian contains new soft SUSY-breaking terms, which include the trilinear parameters $A_{\lambda}$ and $A_{\kappa}$, and the soft mass parameter for the singlet, $m_{S}$. After EW symmetry breaking takes place, the neutral components of the Higgs fields $H_{u, d}$ and the singlet $S$ acquire non-vanishing vacuum expectation values, $v_{u, d}$ and $v_{s}$, respectively. Consequently, an effective term $\mu=\lambda v_{s}$ is generated which is naturally of the order of the EW scale. In terms of the field content, the singlet mixes with the doublet Higgs 
states, giving rise to three CP-even $\left(h_{1}^{0}, h_{2}^{0}, h_{3}^{0}\right)$ and two CP-odd $\left(a_{1}^{0}, a_{1}^{0}\right)$ states, whereas the singlino mixes with the neutralinos, inducing a fifth eigenstate $\left(\widetilde{\chi}_{1}^{0}, \ldots, \widetilde{\chi}_{5}^{0}\right)$, with interesting implications for dark matter searches (see, e.g., refs. [72-79]).

We have carried out a scan with a reduced set of the NMSSM parameters defined at the EW scale, which are detailed in table 1 . The scan is performed with NMSSMTools 3.2.1 [80-82], linked with MultiNest 2.9 [83, 84] to explore the parameter space efficiently. We impose the grand unification relation for the gaugino soft masses, which implies $M_{1}=1 / 2 M_{2}=1 / 6 M_{3}$. Fixed values are used for the trilinear parameters, $A_{t}=1800 \mathrm{GeV}$, $A_{b}=1000 \mathrm{GeV}$, and $A_{\tau}=-1600 \mathrm{GeV}$, as well as for the soft scalar masses of sleptons and squarks, $M_{\widetilde{L}_{i}}=M_{\widetilde{e}_{i}^{c}}=300 \mathrm{GeV}$ and $M_{\widetilde{Q}_{i}}=M_{\widetilde{u}_{i}^{c}}=M_{\widetilde{d}_{i}^{c}}=1000 \mathrm{GeV}$, respectively, where the index $i$ runs over the three families.

We consider the most recent experimental limits on sparticle masses [85-88] derived for simplified SUSY model. For first two generation of squarks we set an optimized lower bound of $1 \mathrm{TeV}$ consistent with refs. [87, 88]. In the same way, the lower limit on lightest stop mass is set to be $m_{\tilde{t}_{1}}>650 \mathrm{GeV}$, while for the lightest sbottom we impose $m_{\tilde{b}_{1}}>700 \mathrm{GeV}[87,88]$. Regarding the gluino mass, we consider $m_{\tilde{g}}>1.2 \mathrm{TeV}$, which holds when the lightest supersymmetric particle (LSP) has non-vanishing mass, and can be independent of squark masses [87]. The situation is far more complicated with EW neutralinos and charginos depending on modes of decay and $m_{\text {LSP }}$. Thus, for lighter chargino we stick to the LEP lower limit of $94 \mathrm{GeV}$ [89]. The lightest neutralino, if light enough, is constrained by its contributions to the invisible $Z$ and Higgs bosons decays. We also take into account experimental bounds on low energy observables [90-104] together with cosmological constraints on the dark matter abundance $[105,106]$ and limits on its spinindependent elastic cross section with quarks from direct detection experiments [107-111]. Regarding the Higgs sector we impose the presence of a SM-like Higgs, which in our case corresponds to the second lightest mass state, in the range $123 \mathrm{GeV} \leq m_{h_{2}^{0}} \leq 127 \mathrm{GeV}$. For the reduced signal strength of the Higgs to di-photon mode, $R_{\gamma \gamma}$, we use $0.23 \leq R_{\gamma \gamma} \leq 1.31$, the latest CMS results at $2 \sigma[22]{ }^{1}$ The remaining reduced signal strengths are also constrained within their respective $2 \sigma$ ranges according to the CMS results of ref. [22] (see refs. [21, 113] for the equivalent ATLAS results). A bound on the branching ratio for invisible Higgs decay [114-121] i.e., $\mathrm{BR}\left(h_{\mathrm{SM}} \rightarrow \widetilde{\chi}_{1}^{0} \widetilde{\chi}_{1}^{0}\right)$ has also been considered in our analysis. Notice that imposing these measurements indirectly entails a strong bound on the non-standard decay modes of the SM-like Higgs boson [118], which in our case affects $\mathrm{BR}\left(h_{2}^{0} \rightarrow h_{1}^{0} h_{1}^{0}\right)$ and $\mathrm{BR}\left(h_{2}^{0} \rightarrow a_{1}^{0} a_{1}^{0}\right)$.

Some low-energy observables also have an important impact in the allowed regions of the NMSSM. We have implemented the recent measurement of the branching ratio of the $B_{s} \rightarrow \mu^{+} \mu^{-}$process by the LHCb [97] and CMS [98] collaboration, which collectively yields $1.5 \times 10^{-9}<\operatorname{BR}\left(B_{s} \rightarrow \mu^{+} \mu^{-}\right)<4.3 \times 10^{-9}$ at $95 \%$ CL [99]. For the $b \rightarrow s \gamma$ decay, we require the $2 \sigma$ range $2.89 \times 10^{-4}<\mathrm{BR}(b \rightarrow s \gamma)<4.21 \times 10^{-4}$, which takes into account theoretical and experimental uncertainties added in quadrature [95, 122125]. We also impose $0.85 \times 10^{-4}<\operatorname{BR}\left(B^{+} \rightarrow \tau^{+} \nu_{\tau}\right)<2.89 \times 10^{-4}$ [94]. We do not

\footnotetext{
${ }^{1}$ For ATLAS the same limit including all systematics is $0.95 \leq R_{\gamma \gamma} \leq 2.55[112,113]$.
} 


\begin{tabular}{|c|c|}
\hline Parameter & Range \\
\hline $\tan \beta$ & $3-20$ \\
$\lambda$ & $0.1-0.7$ \\
$\kappa$ & $0.01-0.6$ \\
$A_{\lambda}$ & $0-1000$ \\
$A_{\kappa}$ & $-100-100$ \\
$\mu$ & $110-300$ \\
$M_{1}$ & $200-500$ \\
\hline
\end{tabular}

Table 1. Ranges of variation in the seven parameters used in the scan. Masses and trilinear terms are given in $\mathrm{GeV}$ units. All the parameters are defined at the EW scale.

impose any constraint on the SUSY contribution to the muon anomalous magnetic moment, $a_{\mu}^{\text {SUSY }}$. As already emphasized in refs. $[126,127]$, the regions of the parameter space that lead to a $125 \mathrm{GeV}$ Higgs generally result in a small $a_{\mu}^{\mathrm{SUSY}}$ which is in tension with experimental results using $e^{+} e^{-}$experimental data [100, 104]. However, if tau data is used, the discrepancy is smaller [103].

We finally require the lightest neutralino to be the LSP and set an upper bound on its relic abundance, $\Omega_{\widetilde{\chi}_{1}^{0}} h^{2}<0.13$, consistent with the latest Planck results [106]. We also impose limits on the spin-independent neutralino-nucleon scattering cross section using the most recent experimental results [107-111].

In our scan, we have built a likelihood function, whose parameters are the neutralino relic density and $m_{h_{2}^{0}}$, which are taken as gaussian probability distribution functions around the measured values with $2 \sigma$ deviations. The lightest pseudoscalar mass is also incorporated in the likelihood in such a way that masses below $m_{a_{1}^{0}}<20 \mathrm{GeV}$ are favoured (although heavier masses are not excluded). This likelihood function is used by MultiNest 2.9 to generate Markov Chain Monte Carlo (MCMC) chains and find regions of the parameter space which maximize the likelihood. Using MultiNest allows us to explore the parameter space of the model more efficiently, since relatively few evaluations are needed to converge to regions which maximize the likelihood.

In figure 1, we show the scatter plot corresponding to different combinations of the input parameters that pass all the experimental constraints. We distinguish two scenarios according to the masses of the Higgs sector. Blue dots correspond to points in the parameter space with $m_{h_{1}^{0}}>m_{h_{2}^{0}} / 2$, while the green ones are those with $m_{h_{1}^{0}}<m_{h_{2}^{0}} / 2$. Since we are interested in neutralino/chargino pair production and its final decay through the $\widetilde{\chi}_{2,3}^{0} \rightarrow \widetilde{\chi}_{1}^{0} a_{1}^{0}$ decay channels, we have denoted with darker colours the points for which $\operatorname{BR}\left(\widetilde{\chi}_{2}^{0} \rightarrow \widetilde{\chi}_{1}^{0} a_{1}^{0}\right)>0.5$ or $\operatorname{BR}\left(\widetilde{\chi}_{3}^{0} \rightarrow \widetilde{\chi}_{1}^{0} a_{1}^{0}\right)>0.5$. On top of this, points in which the mass of the lightest pseudoscalar is lighter than $10 \mathrm{GeV}$ are represented by means of black circles.

In the $(\lambda, \kappa)$ plane (top left panel), points with a lighter $h_{1}^{0}$ (green points) are found in the region of small values of $\kappa$, as in these areas their singlet component is sizable and collider constraints can be avoided. Similarly, very light pseudoscalars accumulate towards small values of $\kappa$, and $A_{\kappa}$, as it can be seen in the top right panel, where the $\left(\kappa, A_{\kappa}\right)$ plane is represented. The presence of a very light pseudoscalar Higgs in the NMSSM requires a 

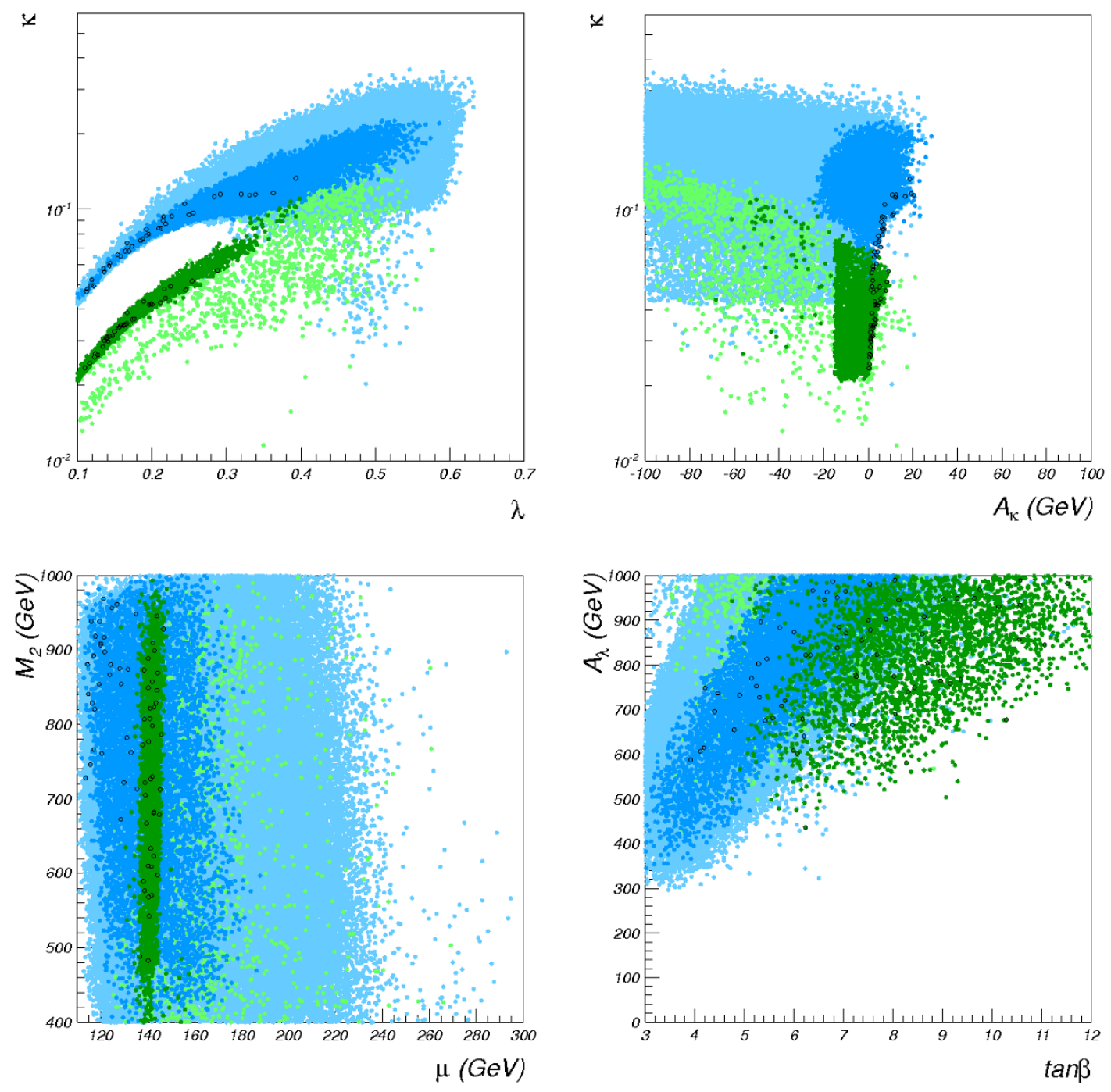

Figure 1. Scatter plot of the allowed regions in the different parameters used as inputs for the scan after all experimental constraints are included. Blue dots represent points for which $m_{h_{1}^{0}}>m_{h_{2}^{0}} / 2$, whereas green dots are those in which $m_{h_{1}^{0}}<m_{h_{2}^{0}} / 2$. Darker points correspond to solutions for which $\operatorname{BR}\left(\widetilde{\chi}_{2}^{0} \rightarrow \widetilde{\chi}_{1}^{0} a_{1}^{0}\right)>0.5$ or $\operatorname{BR}\left(\widetilde{\chi}_{3}^{0} \rightarrow \widetilde{\chi}_{1}^{0} a_{1}^{0}\right)>0.5$. On top of this, black circles correspond to points with $m_{a_{1}^{0}}<10 \mathrm{GeV}$.

tuning of some of the parameters so that either the $\mathrm{U}(1)_{R}$ or $\mathrm{U}(1)_{P Q}$ symmetry of the model is recovered and then this light pseudoscalar would correspond to the pseudo-Goldstone boson of the symmetry [74, 128]. In our scan, the smaller values of the pseudoscalar mass are obtained when $\kappa, A_{\kappa} \rightarrow 0$, for which the $\mathrm{U}(1)_{P Q}$ symmetry is quasi-restored [128].

In the $\left(\mu, M_{2}\right)$ plane (bottom left panel), we can observe that in general the $\mu$ parameter is small. For most of the points, the hierarchical structure $\mu \lesssim M_{1}<M_{2}$ is obtained. One should note that if gaugino universality at the grand unified theory scale is assumed, all points below $M_{2}<400 \mathrm{GeV}$ are excluded by the LHC lower limit on gluino mass. However, we have verified that if the universality relation between $M_{2}$ and $M_{3}$ is broken (setting 

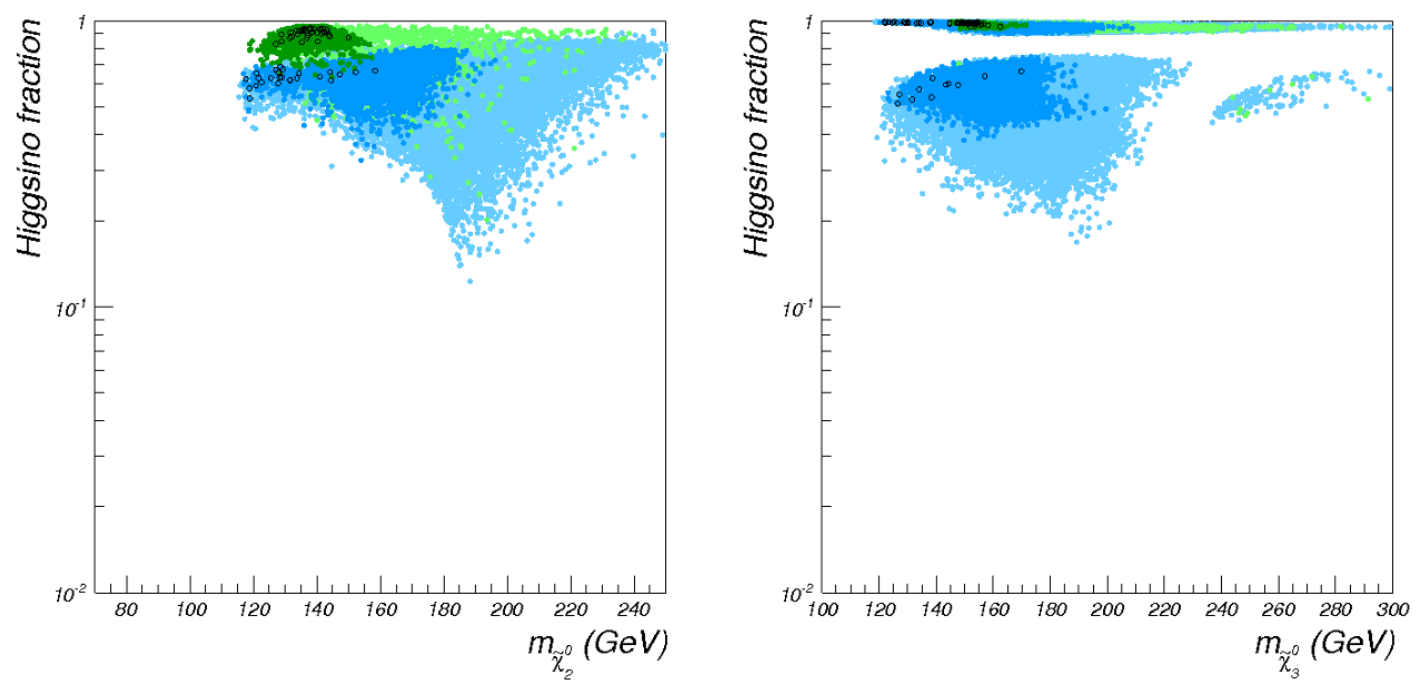

Figure 2. Higgsino fraction of $\widetilde{\chi}_{2}^{0}$ (left) and $\widetilde{\chi}_{3}^{0}$ (right) as a function of their mass. The colour code is the same as in figure 1.

$M_{3}$ high so that $m_{\tilde{g}} \geq 1.2 \mathrm{TeV}$ [87]) viable points for $M_{2} \leq 400 \mathrm{GeV}$ can be obtained. This is a consequence of the fact that gluino mass usually appears in higher order calculations.

The lightest neutralino $\widetilde{\chi}_{1}^{0}$ is therefore mostly singlino (as a consequence of the smallness of the $\kappa$ parameter) but with a Higgsino admixture, which helps to raise its mass and increases for the points with $m_{h_{1}^{0}}>m_{h_{2}^{0}} / 2$. The points with a sizable $\operatorname{BR}\left(\widetilde{\chi}_{2,3}^{0} \rightarrow \widetilde{\chi}_{1}^{0} a_{1}^{0}\right)$ occur for small values of the $\mu$ parameter since this leads to Higgsino-like $\widetilde{\chi}_{2,3}^{0}$ and $\widetilde{\chi}_{1}^{ \pm}$. Although $M_{2}$ is generally large in the scenario with heavier $h_{1}^{0}$, it can be as small as $\sim 300 \mathrm{GeV}$ in the cases with $m_{h_{1}^{0}}<m_{h_{2}^{0}} / 2$. Since this also implies a small $M_{1}$, the second and third lightest neutralinos can also have a non-negligible bino composition. The Higgsino fractions of $\widetilde{\chi}_{2}^{0}$ and $\widetilde{\chi}_{3}^{0}$ are plotted as a function of their mass in figure 2 , where we observe that a large population of points in the parameter space favours Higgsino-like $\widetilde{\chi}_{2,3}^{0}$.

Finally, in the $\left(A_{\lambda}, \tan \beta\right)$ plane, we can see that small values of $\tan \beta(\lesssim 12)$ are preferred for the range used in $A_{\lambda}$. This is useful in order to avoid constraints on some flavour observables, such as $\operatorname{BR}\left(B_{s}^{0} \rightarrow \mu^{+} \mu^{-}\right)$.

In figure 3 , we represent the resulting $\operatorname{BR}\left(\widetilde{\chi}_{2,3}^{0} \rightarrow \widetilde{\chi}_{1}^{0} a_{1}^{0}\right)$ as a function of the mass difference $m_{\widetilde{\chi}_{2,3}^{0}}-\left(m_{h_{1}^{0}}+m_{\widetilde{\chi}_{1}^{0}}\right)$. The alternative decay $\widetilde{\chi}_{2,3}^{0} \rightarrow \widetilde{\chi}_{1}^{0} h_{1}^{0}$ is kinematically open for $m_{\widetilde{\chi}_{2,3}^{0}}-\left(m_{h_{1}^{0}}+m_{\widetilde{\chi}_{1}^{0}}\right)>0$. When this happens, it generally dominates the neutralino decay width and we obtain small values for $\operatorname{BR}\left(\widetilde{\chi}_{2,3}^{0} \rightarrow \widetilde{\chi}_{1}^{0} a_{1}^{0}\right)$. This is a consequence of a relative sign in the corresponding couplings for our choice of signs for $\lambda$ and $\kappa$ [35]. In the scenario with $m_{h_{1}^{0}}<m_{h_{2}^{0}} / 2$, this condition is particularly constraining since the CP-even Higgs is lighter, and for this reason we obtain less viable points in this scenario (green points). Still, we found some solutions featuring $\operatorname{BR}\left(\widetilde{\chi}_{2}^{0} \rightarrow \widetilde{\chi}_{1}^{0} a_{1}^{0}\right)>0.5$, even when the pseudoscalar is very light. On the contrary, points with heavy $h_{1}^{0}$ (blue points) are more easily obtained.

As stated earlier, the presence of light $a_{1}^{0}, h_{1}^{0}$, and $\widetilde{\chi}_{1}^{0}$ can induce non-standard Higgs decays. This is particularly important in the regions of the parameter space with $m_{h_{1}^{0}}<$ 

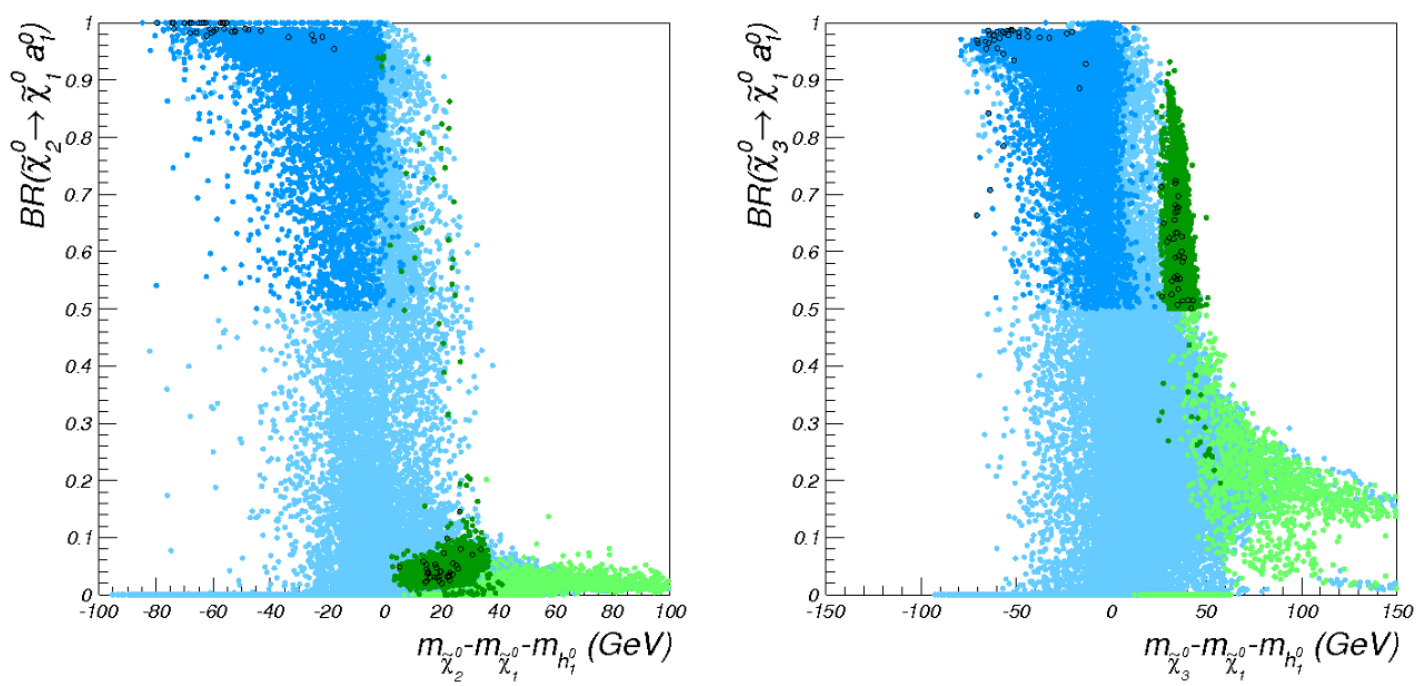

Figure 3. Left: $\operatorname{Br}\left(\widetilde{\chi}_{2}^{0} \rightarrow \widetilde{\chi}_{1}^{0} a_{1}^{0}\right)$ as a function of $m_{\widetilde{\chi}_{2}^{0}}-\left(m_{\widetilde{\chi}_{1}^{0}}+m_{h_{1}^{0}}\right)$. Right: $\operatorname{Br}\left(\widetilde{\chi}_{3}^{0} \rightarrow \widetilde{\chi}_{1}^{0} a_{1}^{0}\right)$ versus $m_{\widetilde{\chi}_{3}^{0}}-\left(m_{\widetilde{\chi}_{1}^{0}}+m_{h_{1}^{0}}\right)$. The colour code is the same as in figure 1 .

$m_{h_{2}^{0}} / 2$ (green points in our plots), since such a light singlet $h_{1}^{0}$ is typically associated with a light singlino-like $\widetilde{\chi}_{1}^{0}$. Thus, all three decay modes $h_{2}^{0} \rightarrow \widetilde{\chi}_{1}^{0} \widetilde{\chi}_{1}^{0}, h_{1}^{0} h_{1}^{0}, a_{1}^{0} a_{1}^{0}$ remain kinematically open for this scenario. In the left plot of figure 4 we have represented the resulting $\operatorname{BR}\left(h_{2}^{0} \rightarrow h_{1}^{0} h_{1}^{0}, a_{1}^{0} a_{1}^{0}\right)$ versus $\operatorname{BR}\left(h_{2}^{0} h_{2}^{0} \rightarrow \widetilde{\chi}_{1}^{0} \widetilde{\chi}_{1}^{0}\right)$. The two contributions $\operatorname{BR}\left(h_{2}^{0} \rightarrow h_{1}^{0} h_{1}^{0}\right)$ and $\operatorname{BR}\left(h_{2}^{0} \rightarrow a_{1}^{0} a_{1}^{0}\right)$ are plotted separately in the right plot of figure 4 . The constraints on the reduced signal strengths of the $h_{2}^{0}$ decays imply an indirect bound $\operatorname{BR}\left(h_{2}^{0} \rightarrow h_{1}^{0} h_{1}^{0}\right)+$ $\mathrm{BR}\left(h_{2}^{0} \rightarrow a_{1}^{0} a_{1}^{0}\right)+\mathrm{BR}\left(h_{2}^{0} h_{2}^{0} \rightarrow \widetilde{\chi}_{1}^{0} \widetilde{\chi}_{1}^{0}\right) \lesssim 0.55$. This upper value is relatively high since we are allowing $2 \sigma$ deviations in all the reduced signal strengths (and in particular on $R_{b b}$ ). Notice also that both $\mathrm{BR}\left(h_{2}^{0} \rightarrow h_{1}^{0} h_{1}^{0}\right)$ and $\mathrm{BR}\left(h_{2}^{0} \rightarrow a_{1}^{0} a_{1}^{0}\right)$ can be sizable and typically dominate over the invisible decay $h_{2}^{0} \rightarrow \widetilde{\chi}_{1}^{0} \widetilde{\chi}_{1}^{0}$.

Finally, in figure 5, we represent the values of the lightest CP-even Higgs mass versus the lightest CP-odd Higgs mass. Notice that there is a large population of points in a square region in the upper right corner. This area satisfies $m_{h_{1}^{0}}>m_{h_{2}^{0}} / 2$ and $m_{a_{1}^{0}}>m_{h_{2}^{0}} / 2$, and therefore, $h_{1}^{0}$ and $a_{1}^{0}$ do not alter the branching ratios of the SM-like Higgs. Outside of this region the constraints on the properties of a SM-like $h_{2}^{0}$ are very stringent. When we demand a very light pseudoscalar and a sizable $\operatorname{BR}\left(\widetilde{\chi}_{2,3}^{0} \rightarrow \widetilde{\chi}_{1}^{0} a_{1}^{0}\right)$, two classes of scenarios are left, those with $m_{h_{1}^{0}} \sim 100 \mathrm{GeV}$ and others with $m_{h_{1}^{0}} \lesssim 60 \mathrm{GeV}$.

In order to proceed with the collider analysis, we have chosen two points in the parameter space which are representative of the different patterns of the Higgs spectrum considered in our work. In particular, the benchmark point BP1 corresponds to an example in which the lightest CP-even Higgs has a mass $m_{h_{1}^{0}} \sim 98 \mathrm{GeV}$, whereas the other benchmark point BP2 illustrates a case with $m_{h_{1}^{0}}<m_{h_{2}^{0}} / 2$. The input values of the NMSSM parameters defining these points are given in table 2 , together with the corresponding mass spectrum. ${ }^{2}$

\footnotetext{
${ }^{2}$ Out of convenience, the value of the top trilinear parameter $A_{t}$ in these benchmark points is slightly different from the fixed one used for the scan. However, this does not affect our conclusions.
} 

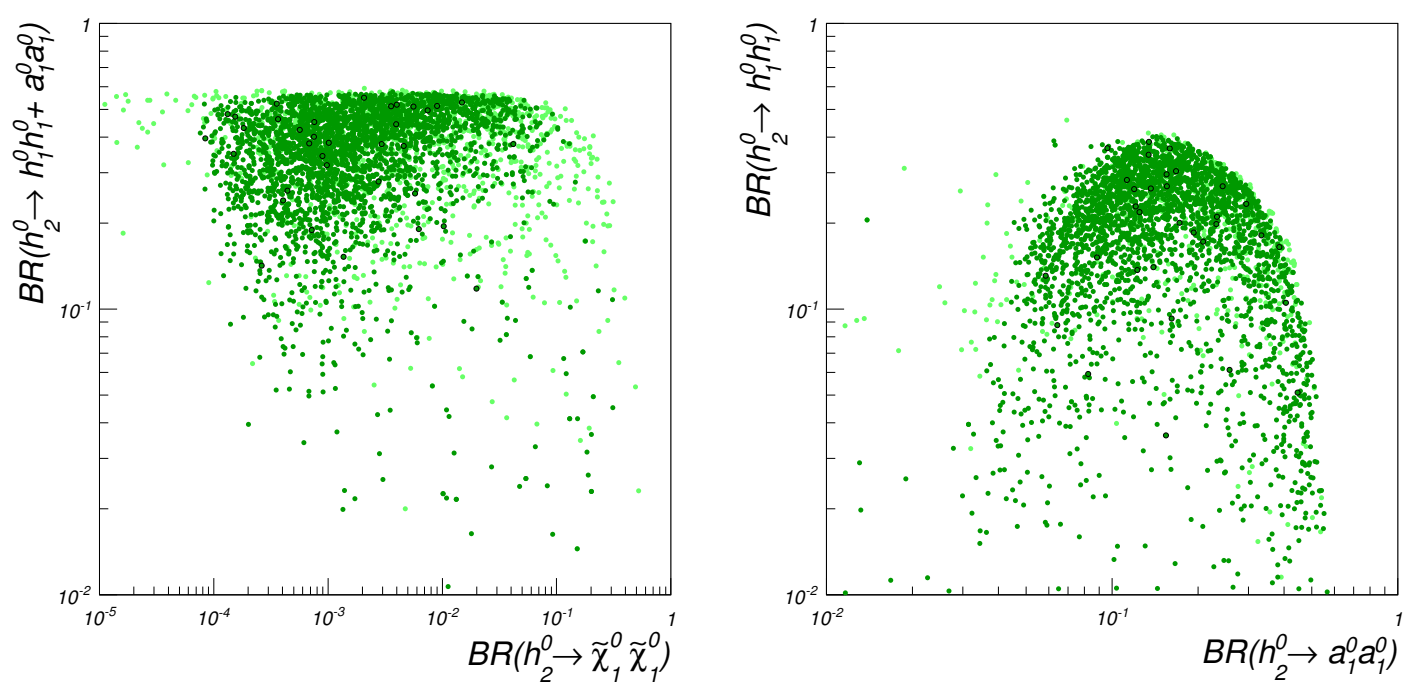

Figure 4. Left: scatter plot of $\operatorname{BR}\left(h_{2}^{0} \rightarrow h_{1}^{0} h_{1}^{0}\right)+\operatorname{BR}\left(h_{2}^{0} \rightarrow a_{1}^{0} a_{1}^{0}\right)$ versus $\operatorname{BR}\left(h_{2}^{0} \rightarrow \widetilde{\chi}_{1}^{0} \widetilde{\chi}_{1}^{0}\right)$. Right: scatter plot of $\mathrm{BR}\left(h_{2}^{0} \rightarrow h_{1}^{0} h_{1}^{0}\right)$ versus $\mathrm{BR}\left(h_{2}^{0} \rightarrow a_{1}^{0} a_{1}^{0}\right)$. The colour code is the same as in figure 1 but here we only consider the scenario with $m_{h_{1}^{0}}<m_{h_{2}^{0}} / 2$.

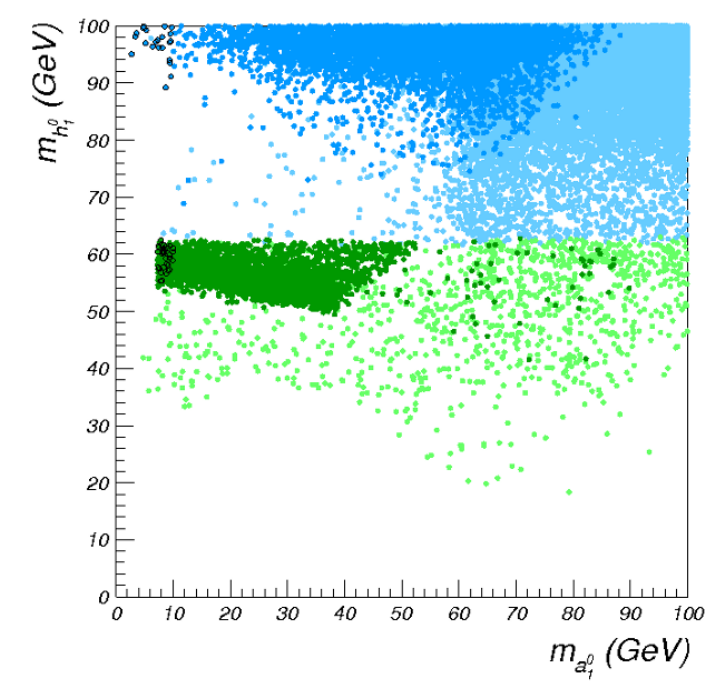

Figure 5. Lightest CP-even Higgs mass versus the lightest CP-odd Higgs mass. The color code is the same as in figure 1.

These points are similar to those studied in a previous analysis [26] in the context of scalar Higgs decays $h \rightarrow a_{1}^{0} a_{1}^{0}$. It should be noted that in order to obtain such a light $a_{1}^{0}$ the input parameters have to be carefully tuned.

In both benchmark points (BP1, BP2) the lightest neutralino, $\widetilde{\chi}_{1}^{0}$ is mainly singlet-like $(56.2 \%, 66.9 \%)$ with a sizable Higgsino composition $(43.3 \%, 30.5 \%)$. Regarding $\widetilde{\chi}_{2,3}^{0}$ and $\tilde{\chi}_{1}^{ \pm}$, they are mostly Higgsino and light (due to the smallness of the $\mu$ parameter and the relatively large values of $M_{1}$ and $M_{2}$ ), and thus their productions are greatly enhanced.

The pair production of Higgsino-like $\widetilde{\chi}_{2}^{0}, \widetilde{\chi}_{3}^{0}$, and $\widetilde{\chi}_{1}^{ \pm}$can lead to multi-lepton final states through decay chains in which very light pseudoscalars are produced. More specifically, in 


\begin{tabular}{|c|c|c|}
\hline & $\mathrm{BP} 1$ & $\mathrm{BP} 2$ \\
\hline $\tan \beta$ & 5 & 5 \\
$\lambda, \kappa$ & $0.285,0.114$ & $0.286,0.0844$ \\
$A_{\lambda}, A_{\kappa}$ & $660,13.8$ & $820,14.35$ \\
$M_{\widetilde{L}_{i}}, M_{\widetilde{e}_{i}^{c}}$ & 300,300 & 300,300 \\
$M_{\widetilde{Q}_{i}}, M_{\widetilde{u}_{i}^{c}}, M_{\widetilde{d}_{i}^{c}}$ & $1000,1000,1000$ & $1000,1000,1000$ \\
$\mu$ & 123.0 & 123.5 \\
$M_{1}, M_{2}, M_{3}$ & $480,960,2880$ & $250,500,1500$ \\
$A_{\tau}, A_{b}, A_{t}$ & $-1600,1000,1850$ & $-1600,1000,1250$ \\
\hline$m_{h_{1}^{0}}, m_{h_{2}^{0}}, m_{h_{3}^{0}}$ & $97.7,125.5,662.4$ & $62.0,125.6,739.6$ \\
$m_{a_{1}^{0}}, m_{a_{2}^{0}}$ & $6.3,660.8$ & $7.6,738.4$ \\
$m_{h^{ \pm}}, m_{\widetilde{\chi}_{1}^{ \pm}}, m_{\widetilde{\chi}_{2}^{ \pm}}$ & $664.3,122.6,965.2$ & $738.8,118.1,522.3$ \\
$m_{\widetilde{\chi}_{1}^{0}}, m_{\widetilde{\chi}_{2}^{0}}, m_{\widetilde{\chi}_{3}^{0}}$ & $84.4,136.0,140.9$ & $63.8,125.3,139.0$ \\
$m_{\widetilde{t}_{1}}, m_{\widetilde{t}_{2}}, m_{\widetilde{b}_{1}}, m_{\widetilde{b}_{2}}$ & $644.0,1048.5,858.7,861.3$ & $950.6,1134.9,1037.0,1038.6$ \\
$m_{\widetilde{\tau}_{1}}, m_{\widetilde{\tau}_{2}}$ & $296.5,309.5$ & $296.5,309.5$ \\
$m_{\widetilde{g}}$ & 2768.4 & 1496.0 \\
\hline
\end{tabular}

Table 2. Model parameters that define our choice of benchmark points and resulting spectrum. The top-quark pole mass is set to $173.5 \mathrm{GeV}$ and $m_{b}{ }^{\overline{\mathrm{MS}}}\left(m_{b}\right)=4.18 \mathrm{GeV}$. All the masses are given in $\mathrm{GeV}$.

\begin{tabular}{|c|c|c|}
\hline & $\mathrm{BP} 1$ & $\mathrm{BP} 2$ \\
\hline $\operatorname{Br}\left(\widetilde{\chi}_{1}^{ \pm} \rightarrow \ell^{ \pm} \nu_{\ell} \widetilde{\chi}_{1}^{0}\right)$ & 0.11 & 0.11 \\
$\operatorname{Br}\left(\widetilde{\chi}_{2}^{0} \rightarrow \widetilde{\chi}_{1}^{0} a_{1}^{0}\right)$ & 1.00 & 1.00 \\
$\operatorname{Br}\left(\widetilde{\chi}_{3}^{0} \rightarrow \widetilde{\chi}_{1}^{0} a_{1}^{0}\right), \operatorname{Br}\left(\widetilde{\chi}_{3}^{0} \rightarrow \widetilde{\chi}_{2}^{0} a_{1}^{0}\right)$ & $0.98,0.0$ & $0.76,0.20$ \\
\hline $\operatorname{Br}\left(a_{1}^{0} \rightarrow \tau^{+} \tau^{-}\right)$ & 0.93 & 0.92 \\
\hline
\end{tabular}

Table 3. Relevant branching fractions for multi-lepton search channels in the two benchmark points.

the scenarios under study, the second and third lightest neutralinos decay as, $\widetilde{\chi}_{2,3}^{0} \rightarrow \widetilde{\chi}_{1}^{0}+a_{1}^{0}$, $\widetilde{\chi}_{3}^{0} \rightarrow \widetilde{\chi}_{2}^{0}+a_{1}^{0} \rightarrow \widetilde{\chi}_{1}^{0}+2 a_{1}^{0}$, and for the range of masses considered $\left(2 m_{\tau}<m_{a_{1}^{0}}<2 m_{b}\right)$, the lightest pseudoscalar predominantly decays into a pair of taus, $a_{1}^{0} \rightarrow \tau^{+} \tau^{-}$. Notice that this differs from conventional analysis, in which sleptons or $Z$ intermediate states are involved in lepton production. On the other hand, the lighter chargino, $\widetilde{\chi}_{1}^{ \pm}$, mainly decays through slepton or sneutrino mediated standard modes into $\widetilde{\chi}_{1}^{0} \ell^{ \pm} \nu_{\ell}$. The corresponding branching ratios for these processes can be found in table 3 , where we can observe that they are sizable in both benchmark points. 


\begin{tabular}{|r|r|r|}
\hline & $\mathrm{BP} 1$ & $\mathrm{BP} 2$ \\
\hline$\sigma_{\widetilde{\chi}_{3}^{0} \widetilde{\chi}_{1}^{0}+\sigma_{\widetilde{\chi}_{3}^{0} \widetilde{\chi}_{2}^{0}}+\sigma_{\widetilde{\chi}_{3}^{0} \widetilde{\chi}_{3}^{0}}} 185.8$ & 491.9 \\
$\sigma_{\widetilde{\chi}_{3}^{0} \widetilde{\chi}_{1}^{ \pm}}$ & 437.2 & 729.1 \\
$\sigma_{\widetilde{\chi}_{2}^{0} \widetilde{\chi}_{1}^{0}}+\sigma_{\widetilde{\chi}_{2}^{0} \widetilde{\chi}_{2}^{0}}$ & 309.8 & 4.3 \\
$\sigma_{\widetilde{\chi}_{2}^{0} \widetilde{\chi}_{1}^{ \pm}}$ & 727.2 & 648.7 \\
\hline
\end{tabular}

Table 4. Cross sections in fb calculated with HeRwIG ++ for the direct production of neutralino and chargino pairs at the $8 \mathrm{TeV}$ LHC for our choice of benchmark points.

The signals of interest are therefore

$$
\begin{aligned}
\widetilde{\chi}_{2,3}^{0} \widetilde{\chi}_{1}^{ \pm} & \rightarrow \ell^{+} \ell^{-} \ell^{ \pm}+\mathbb{E}_{\mathrm{T}}, \\
\widetilde{\chi}_{3}^{0} \widetilde{\chi}_{1}^{ \pm} & \rightarrow 2 \ell^{+} 2 \ell^{-} \ell^{ \pm}+\mathbb{E}_{\mathrm{T}}, \\
\tilde{\chi}_{3}^{0} \widetilde{\chi}_{1}^{0} & \rightarrow 2 \ell^{+} 2 \ell^{-}+\mathbb{E}_{\mathrm{T}}, \\
\widetilde{\chi}_{2,3}^{0} \widetilde{\chi}_{2,3}^{0} & \rightarrow 2 \ell^{+} 2 \ell^{-}+\mathbb{E}_{\mathrm{T}}, \\
\widetilde{\chi}_{2}^{0} \widetilde{\chi}_{3}^{0} & \rightarrow 3 \ell^{+} 3 \ell^{-}+\mathbb{E}_{\mathrm{T}}, \\
\widetilde{\chi}_{3}^{0} \widetilde{\chi}_{3}^{0} & \rightarrow 4 \ell^{+} 4 \ell^{-}+\mathbb{E}_{\mathrm{T}} .
\end{aligned}
$$

The missing energy is associated to the lightest neutralino $\widetilde{\chi}_{1}^{0}$ and the neutrinos from $\tau$ or $\tilde{\chi}_{1}^{ \pm}$decays. Since $\operatorname{BR}\left(a_{1}^{0} \rightarrow \tau^{+} \tau^{-}\right) \sim 1$, the fraction of lepton flavors in eq. (2.2) is related to that of tau decays. ${ }^{3}$

Notice that in principle one can also consider neutralino-chargino production from stop decay. In fact, as it was argued in ref. [38], this could be an important production channel if gluinos or stops were light, and $\widetilde{t}_{1} \rightarrow \widetilde{\chi}_{2}^{0} t, \widetilde{\chi}_{3}^{0} t, \widetilde{\chi}_{1}^{ \pm} b$ can be enhanced if the Higgsino components of $\widetilde{\chi}_{2}^{0}, \widetilde{\chi}_{3}^{0}, \widetilde{\chi}_{1}^{ \pm}$are large. These decay chains can give rise to multilepton signals accompanied by hadronic jets and missing energy $\widetilde{t}_{1}{\widetilde{t_{1}^{*}}}_{1} \rightarrow n \ell^{+}+n^{\prime} \ell^{-}+n^{\prime \prime}$ jets $+\mathbb{E}_{\mathrm{T}}$. This signal can be important when LHC starts operating at a higher center of mass energy. However, given the current lower mass bounds on coloured particles from the current $8 \mathrm{TeV}$ LHC results, squark or gluino decays are not generally the main production channels for neutralinos in many points of the parameter space. This statement is generically true even when squark masses are around $1 \mathrm{TeV}$ : although squark pair production can be significant, the cascade decays are generally suppressed by the corresponding branching fractions on each step. Thus, contrary to the analysis of ref. [38], we will only consider neutralino/chargino pair production.

\section{Direct production of neutralinos decaying into a light pseudoscalar}

In this section, we examine how collider signatures of eq. (2.2) can be detected by using dedicated object reconstruction schemes and kinematic variables. The sparticle mass spec-

\footnotetext{
${ }^{3}$ In principle the longer decay mode like $\widetilde{\chi}_{2,3}^{0} \rightarrow \widetilde{\chi}_{1}^{0} h_{1}^{0} \rightarrow \widetilde{\chi}_{1}^{0} a_{1}^{0} a_{1}^{0}$ can also give rise to interesting multilepton final states. In this work, however, we only considered the simplest mode of eq. (2.2) since it already gives good statistical significance.
} 
trum and decay widths for the selected benchmark points are calculated with NMSSMTOOLS, whose output is processed with HERWIG++ 2.6.3 [129, 130], interfaced with CTEQ6L1 parton distribution functions [131], in order to calculate the production cross-sections. For the SUSY signals, all possible pair productions of the light neutralinos $\widetilde{\chi}_{1,2,3}^{0}$ and the charginos $\widetilde{\chi}_{1}^{ \pm}$have been considered. The production cross sections for each benchmark point are given in table 4 , where we can observe that the main products are neutralino-chargino pairs, i.e., $\widetilde{\chi}_{2,3}^{0} \widetilde{\chi}_{1}^{ \pm}$, whereas neutralino pairs $\widetilde{\chi}_{2,3}^{0} \widetilde{\chi}_{2,3}^{0}$ are sub-leading. The differences of the cross section values are mainly due to the singlet compositions of the light neutralinos. For instance, the singlino component of $\widetilde{\chi}_{2}^{0}$ in $\mathrm{BP} 2$ is $\sim 30 \%$, while it is only $\sim 3 \%$ in $\mathrm{BP} 1$, which results in a reduced value of the cross section for neutralino-pair production, $\sigma_{\widetilde{\chi}_{2}^{0} \widetilde{\chi}_{1,2}^{0}}$, in BP2.

In order to study the feasibility of observing the proposed signal at the current configuration of the LHC, we have generated Monte Carlo (MC) event samples of the NMSSM signal of direct neutralino pairs, as well as neutralino-chargino pairs, for a proton-proton collision at the center-of-mass energy of $8 \mathrm{TeV}$ using HERWIG++. After performing the parton showering and the hadronization with HeRwiG++, the generator-level MC events have been processed with DeLPHes 3.0.7 [132] using a modified CMS card to obtain the detector-level data. Jets are formed using the anti- $k_{t}$ jet clustering algorithm [133] with the distance parameter of 0.5 . Then, they are required to have a transverse momentum $p_{\mathrm{T}}>20 \mathrm{GeV}$ and a pseudo-rapidity $|\eta|<2.5$ in the analysis. The $b$-tagging efficiency is set to be $70 \%$ for a jet with $p_{\mathrm{T}}>30 \mathrm{GeV}$, while the mis-tagging rates are assumed to be $10 \%$ and $1 \%$ for the $c$-jets and the light-flavor jets, respectively.

For choosing isolated lepton candidates (throughout the text, isolated lepton includes $e, \mu$, and hadronically-decaying $\tau, \tau_{h}$ ), the threshold $p_{\mathrm{T}}$ of $5 \mathrm{GeV}$ is adopted and the scalar sum of the transverse momenta of whole charged tracks with $p_{\mathrm{T}}>0.5 \mathrm{GeV}$ lying in a cone of $\Delta R=0.1$ around the candidate has been calculated (we will later justify the need for such a small $\Delta R$ ). The candidate lepton is accepted as being isolated if the fraction of the sum to the candidate's transverse momentum is less than $10 \%$. This isolated lepton criterion is different from the one used in the ATLAS [134] and CMS [135] analyses to look for the direct neutralino-chargino pair production. For instance, the CMS analysis selects the cone size of 0.3 and the transverse momentum fraction less than $15 \%$, in order to pick up both isolated electrons and muons. A better isolation of the lepton with larger cone size appears at the cost of reducing the number of isolated leptons. However, we find that the criteria adopted by the experimental collaborations often fail to capture the signal leptons since the leptons in the signal of interest are likely to be very close to the other lepton sharing the same parent pseudoscalar and possess relatively soft transverse momentum. When adopting the conventional criterion, we found that the signal is almost hidden in the backgrounds, and for this reason we relax the criterion of the cone size while being more strict on the fraction value. One can further attempt to tune the parameters for the signals of each benchmarks, however it is beyond the scope of our work since a concrete knowledge of the detector performance is necessary for that. Instead of tuning, the fake leptons originated from the jets are removed by imposing separate cuts.

The isolated lepton chosen by the criterion is discarded if its angular separation to the adjacent jet with $p_{\mathrm{T}}>20 \mathrm{GeV}$ is within a range of $\Delta R<0.4$. Furthermore, the 

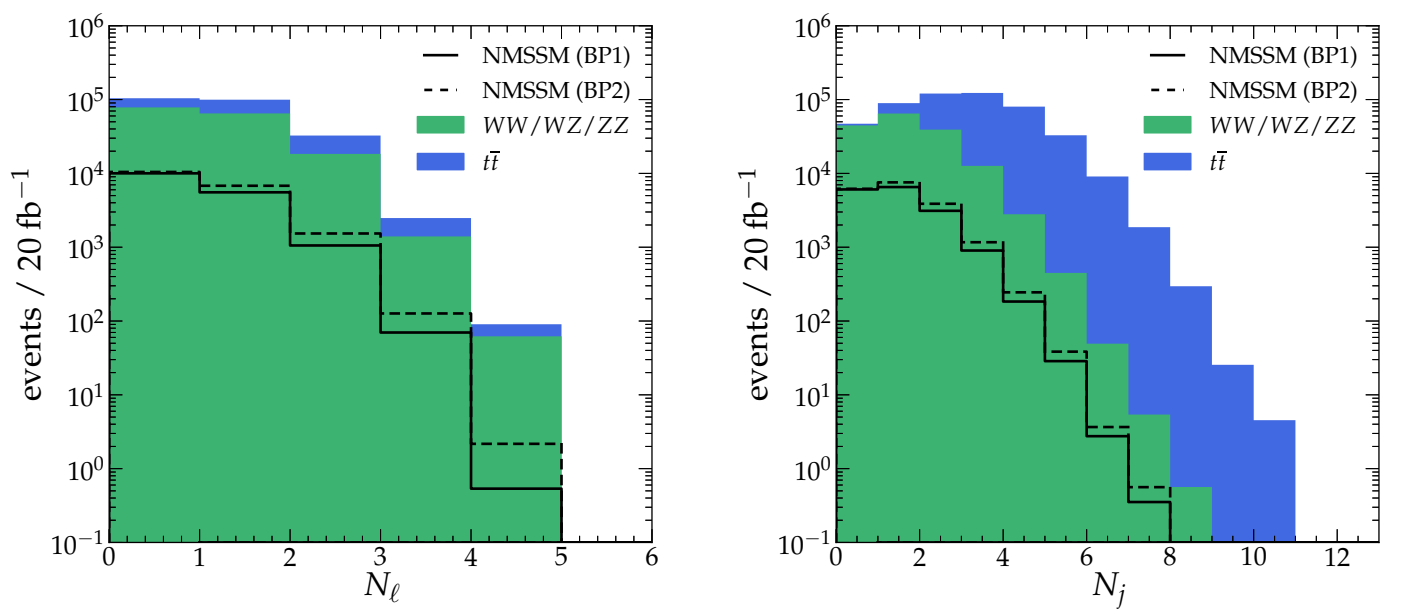

Figure 6. Number of (left panel) charged leptons satisfying $p_{\mathrm{T}}>10 \mathrm{GeV}$ and $|\eta|<2.4$ after choosing the isolated leptons and (right panel) jets with $p_{\mathrm{T}}>20 \mathrm{GeV}$ and $|\eta|<2.5$.

event containing an opposite-sign same-flavor (OSSF) lepton pair with an invariant mass below $12 \mathrm{GeV}$ is rejected to suppress the low-mass continuum backgrounds. This cut also rejects some signal events due to the low-mass pseudoscalar, however, the signals can still pass the cut since they can have different-flavor lepton pairs. Then, the event is selected for the analysis when it has at least one isolated electron or muon with $p_{\mathrm{T}}^{e}>12 \mathrm{GeV}$ or $p_{\mathrm{T}}^{\mu}>8 \mathrm{GeV}$ and $|\eta|<2.4$. Since the efficiency for the tau-jet identification is poor and our analysis largely relies on leptonic tau decays, we use a conventional criterion for reconstructing the tau-jets with a cone size of 0.5 and the minimum $p_{\mathrm{T}}$ value of $10 \mathrm{GeV}$. In the analysis, we select only the tau-jet with $p_{\mathrm{T}}>15 \mathrm{GeV}$. In figure 6 , we show the lepton and jet multiplicity distributions for both signals and backgrounds. The missing transverse momentum $\boldsymbol{p}_{\mathrm{T}}$ is defined as the negative vector sum of the transverse momenta of all the calibrated calorimetric energy clusters and muon candidates. In SUSY signals, the main source of the missing energy is the neutrinos from the decays of taus or charginos, as well as the undetectable neutralino LSP.

The main SM backgrounds consist of the EW diboson ( $W W, W Z, Z Z)$ and triboson $(W W W, W W Z, Z Z Z)$, resulting in leptonic final states, the dileptonic top-pair, $t \bar{t} W / Z$, Drell-Yan (DY), and $Z+$ jet processes. All the background processes except triboson and $t \bar{t} W / Z$, for which MADGraph 5 [136] has been used, are generated by Herwig ++ , interfaced with DELPHES to simulate the detector effects and reconstruct the final-state objects. We use the measured cross section values in the recent CMS analysis results for the most important SM background processes of diboson [137, 138] and dileptonic $t \bar{t}[139]$, while the values calculated with Herwig ++ for the DY and $Z+$ jet and MADGraPH for the triboson and $t \bar{t} W / Z$ processes are used when estimating the backgrounds.

Concerning SUSY backgrounds with conserved $R$-parity, the dilepton invariant mass around $m_{a_{1}^{0}}$ can be a useful separator. On the other hand, since the singlino like $\widetilde{\chi}_{1}^{0}$ can be very light, large missing transverse energy might no longer be a good discriminator to $R$-parity violating models. Especially, non-minimal SUSY models with broken $R$-parity, 


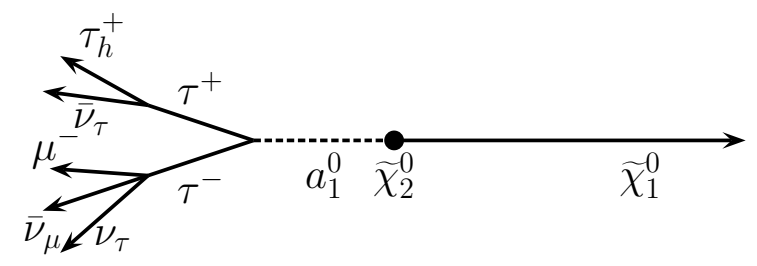

Figure 7. A kinematic configuration of the signal decay events in the rest frame of $\widetilde{\chi}_{2}^{0}$.

e.g., the $\mu \nu \mathrm{SSM}[140,141]$ can accommodate similar light scalars, pseudoscalars and neutralinos $[142,143]$ and hence produces similar final states. However, measurable displaced vertices for the latter class of models could be useful to distinguish among these constructions. Finally, backgrounds arising either from squark/gluino mediated cascades or from decays of heavier neutralino-chargino pair can be isolated from the studied signal in terms of final state lepton and jet multiplicity.

In order to increase the ratio of the signal to the backgrounds, the following basic event selection cuts are applied.

- At least three isolated leptons, $\ell=e, \mu, \tau_{h}$, where $\tau_{h}$ denotes the $\tau$-jet, and at least one of them is required to have $p_{\mathrm{T}}>20 \mathrm{GeV}$.

- No $b$-tagged jet.

- For electrons and muons, the invariant mass of the OSSF leptons $m_{\ell^{+} \ell^{-}}^{\text {OSSF }}$ must satisfy $\left|m_{\ell^{+} \ell^{-}}^{\mathrm{OSSF}}-m_{Z}\right|>15 \mathrm{GeV}$ to exclude the backgrounds associated with the leptonicallydecaying $Z$ boson.

In the latest ATLAS and CMS studies, the neutralinos and charginos are assumed to be practically EW gauginos and the decays are mediated by on/off-shell sleptons or EW gauge bosons like

$$
\widetilde{\chi}_{2}^{0}+\widetilde{\chi}_{1}^{ \pm} \rightarrow \ell^{ \pm} \widetilde{\ell}^{\mp(*)}\left(Z^{(*)} \widetilde{\chi}_{1}^{0}\right)+\ell^{\prime \pm} \nu_{\ell^{\prime}} \widetilde{\chi}_{1}^{0} \rightarrow \ell^{+} \ell^{-} \ell^{\prime \pm}+\mathscr{E}_{\mathrm{T}} \mathrm{.}
$$

In order to interpret the search results, simplified SUSY model points are considered and the missing energy $\mathbb{E}_{\mathrm{T}}$, the dilepton invariant mass $m_{\ell \ell}$, and the transverse mass $M_{\mathrm{T}} \equiv \sqrt{\left(\left|\mathbf{p}_{\mathrm{T}}^{\ell}\right|+\mathscr{E}_{\mathrm{T}}\right)^{2}-\left|\mathbf{p}_{\mathrm{T}}^{\ell}+\mathbf{p}_{\mathrm{T}}\right|^{2}}$ are employed as the main kinematic variables. In our benchmark scenarios, the final state can be similar to that in the simplified models, however, the search strategy should be basically different from those studies not only because of the fact that the Higgsino-like neutralinos $\widetilde{\chi}_{3}^{0}$ as well as $\widetilde{\chi}_{2}^{0}$ come into play in the SUSY signal productions, but also because of the existence of the light pseudoscalar that decays predominantly into a pair of tau leptons. In particular, due to the light pseudoscalar, the taus are nearly collinear and the visible final state particles become relatively soft since a portion of tau energy is carried away by the neutrinos. In figure 7 , one can see an example of the kinematic configuration in the rest frame of $\widetilde{\chi}_{2}^{0}$. The kinematic configuration often results in the failure of reconstructing the $\tau$-jet, while the electron or muon can still 


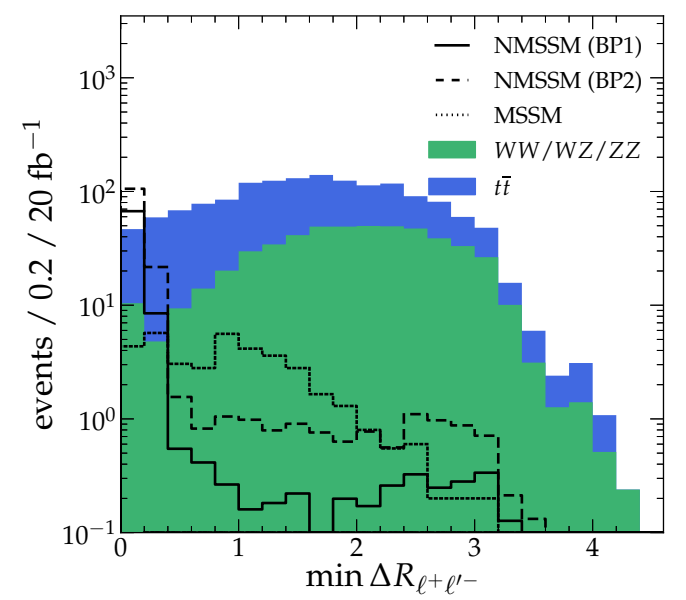

Figure 8. Distributions of the smallest $\Delta R_{\ell^{+} \ell^{\prime-}}$ The basic event selection cuts are applied for both signals and backgrounds.

have chances to be identified as the isolated lepton. If at least two isolated leptons $(e, \mu$ or $\tau$-jet), sharing the same parent pseudoscalar $a_{1}^{0}$, are successfully identified, their angular separation $\Delta R_{\ell^{+} \ell^{\prime-}} \equiv \sqrt{\left(\Delta \phi_{\ell^{+} \ell^{\prime-}}\right)^{2}+\left(\Delta \eta_{\ell^{+} \ell^{\prime-}}\right)^{2}}$ will turn out to be small and can be estimated as

$$
\Delta R_{\ell^{+} \ell^{\prime}-} \sim \frac{4 m_{\widetilde{\chi}_{i}^{0}} m_{a_{1}^{0}}}{m_{\widetilde{\chi}_{i}^{0}}^{2}-m_{\widetilde{\chi}_{j}^{0}}^{2}},
$$

in the case of $\widetilde{\chi}_{i}^{0} \rightarrow \widetilde{\chi}_{j}^{0} a_{1}^{0} \rightarrow \widetilde{\chi}_{j}^{0} \tau^{+} \tau^{-}$. Since our basic event selection cuts demand that there are at least three leptons in the event, all possible combinations of the opposite-sign leptons are considered, and then the smallest value of $\Delta R_{\ell^{+} \ell^{\prime}}$ is chosen. Both signal and dominant background distributions are shown in figure 8 .

A similar final state can arise in a simplified MSSM with the sparticle mass hierarchy of $m_{\widetilde{\chi}_{1}^{0}}<m_{\widetilde{\tau}}<m_{\widetilde{\chi}_{2}^{0}}<m_{\widetilde{e}, \widetilde{\mu}}$. In this case, the taus will be produced by the mediation of the stau. This corresponds to the tau-dominated scenario in the CMS analysis [135]. For a comparison, we pick up one MSSM sample point, calculated with SOFTSUSY 3.3.5 [144], whose mass spectrum is the similar as our NMSSM benchmark points except $m_{\widetilde{\tau}} \approx\left(m_{\widetilde{\chi}_{1}^{0}}+\right.$ $\left.m_{\widetilde{\chi}_{2}^{0}}\right) / 2$, and then produce the detector-level events by HerwiG ++ and Delphes. Since the mediating stau is a scalar state, the angular separation among the final-state leptons is not expected to be confined in the small $\Delta R_{\ell^{+} \ell^{\prime}}$ region aside from the error due to the mis-paired leptons. Figure 8 demonstrates that the condition with the collinear leptons is not useful in the typical MSSM point.

Although the $m_{\ell^{+} \ell^{-}}^{\text {OSSF }}$ cut removes the background processes associated with $Z$ bosons at least partially, $Z \rightarrow \tau^{+} \tau^{-} \rightarrow \ell^{+} \ell^{\prime-}+\mathbb{E}_{\mathrm{T}}$ events can be further reduced by imposing a cut on dilepton invariant masses for all possible combinations of the isolated leptons $(e, \mu$ and $\tau$-jet), including opposite-sign different-flavor (OSDF) lepton pairs. The left panel of figure 9 shows a peak structure around the $Z$ boson mass in the background distribution, while the SUSY signals are populated largely in the region of the small mass value. This observation encourages us to use selection cuts as follows. 

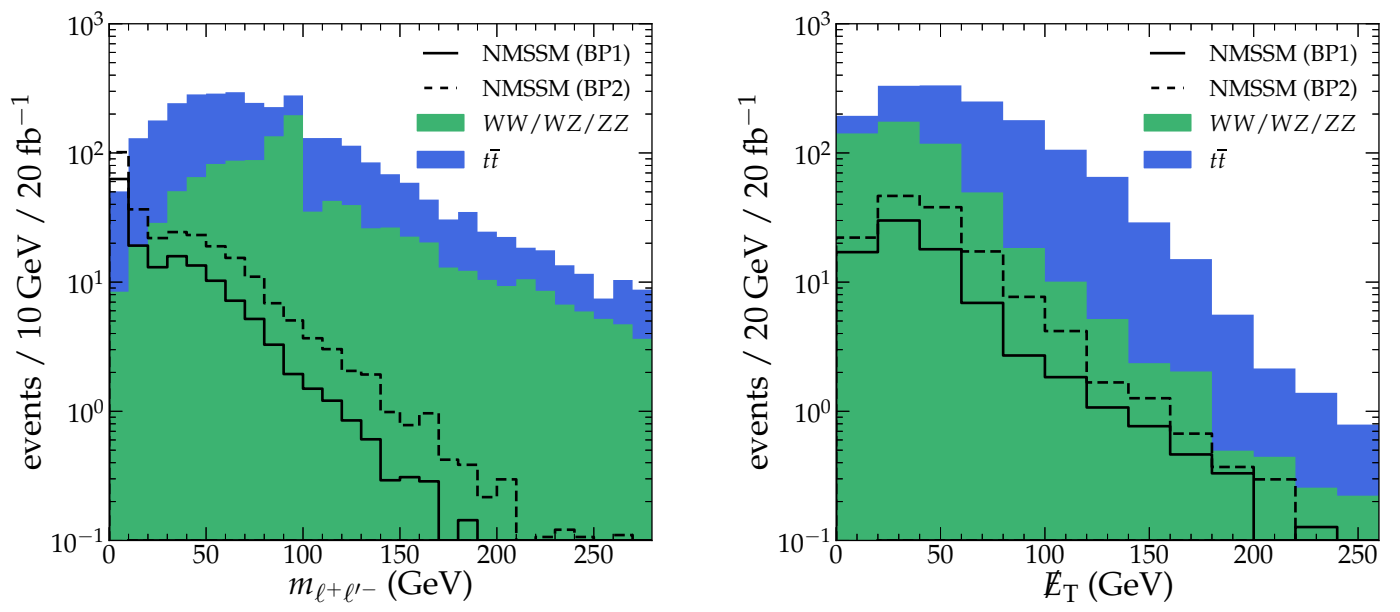

Figure 9. Distributions of (left panel) dilepton invariant mass for all possible combinations of opposite-sign isolated leptons and (right panel) the missing energy. Background distributions are normalized to match the signal distributions. The basic event selection cuts are applied for both signals and backgrounds.

- If any $m_{\ell^{+} \ell^{\prime}}$ for $\ell, \ell^{\prime}=e, \mu, \tau_{h}$ satisfies $\left|m_{\ell^{+} \ell^{\prime-}}-m_{Z}\right|<20 \mathrm{GeV}$, the event is discarded.

- $\min _{\forall\left\{\ell^{+} \ell^{\prime}\right\}}\left\{m_{\ell^{+} \ell^{\prime}}\right\}<10 \mathrm{GeV}$.

The latter cut is set to ensure that at least one pair of leptons originates from the light pseudoscalar. Since similar cuts on $m_{\ell^{+} \ell^{-}}^{\mathrm{OSSF}}$ have already been imposed in the basic selection of events, the cut conditions above are practically applied to the OSDF lepton pairs.

As mentioned above, the recent searches for the SUSY signature at the LHC have employed kinematic variables like the missing energy $\mathbb{E}_{\mathrm{T}}$ and the transverse mass $M_{\mathrm{T}}$ as well as the dilepton invariant mass. Strong cuts on these variables can be validated if a heavy LSP pair is the main source of the missing energy and the mass gap among sparticles are large enough so that the visible leptons are very energetic. However, in our benchmark scenarios, the missing energy can be quite small due to the cancellation between the LSP and neutrinos. This is one of distinguishing features of the scenario since the neutrinos from the tau decay are nearly collinear and the sum of the neutrino momenta would cancel partially the LSP momenta in the rest frame of the heavier neutralino as in figure 7 . This can be checked by seeing the distributions of the MC events shown in the right panel of figure 9 . Still, the $\mathbb{E}_{\mathrm{T}}$ cut should be applied to suppress the backgrounds containing little missing energies like in the QCD multi-jet processes faking leptons. We here impose rather a mild cut on the missing energy, $\mathbb{E}_{\mathrm{T}}>30 \mathrm{GeV}$. Moreover, many of the isolated $e, \mu$ in the SUSY signal events are from the tau, which is already quite collinear to the parent light pseudoscalar, the visible lepton would be soft as discussed above. ${ }^{4}$ This situation with the

\footnotetext{
${ }^{4}$ At least one hard isolated lepton can be produced in the leptonic decay process of the lighter chargino, i.e., $\widetilde{\chi}_{1}^{ \pm} \rightarrow \ell^{ \pm} \nu_{\ell} \widetilde{\chi}_{1}^{0}$.
} 

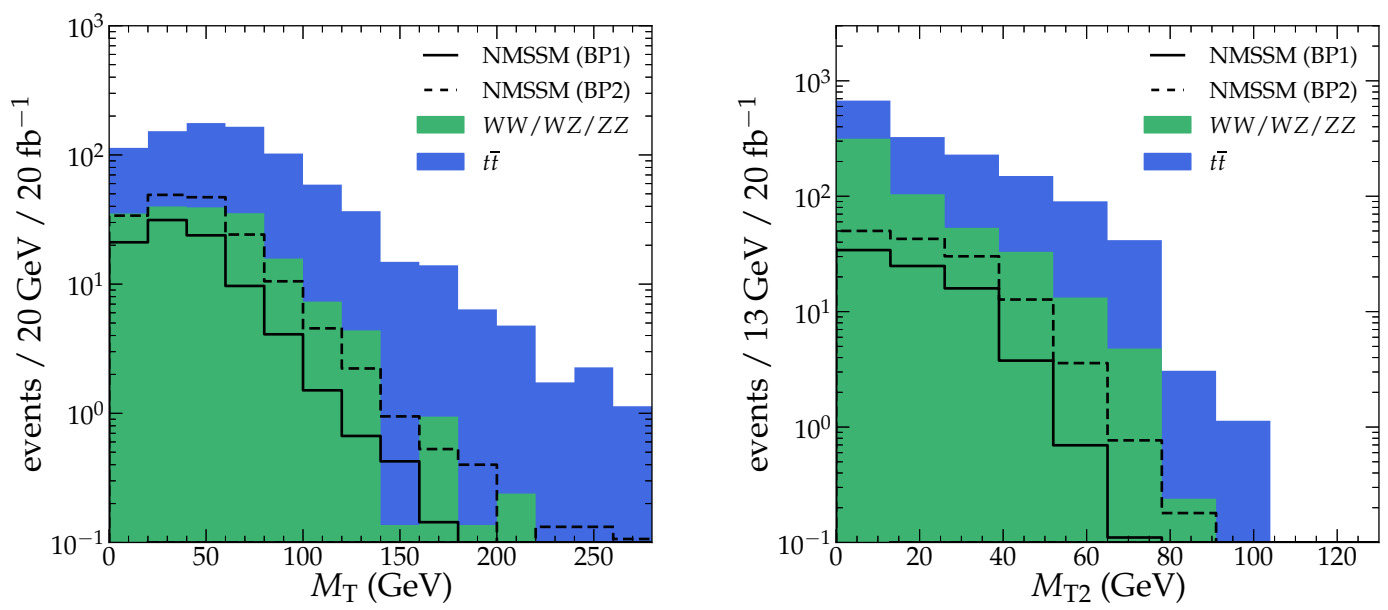

Figure 10. Distributions of (left panel) transverse mass for one lepton + missing energy and (right panel) and the $M_{\mathrm{T} 2}$ for an opposite-sign lepton pair and missing energy. Background distributions are normalized to match the signal distributions. The basic event selection cuts are applied for both signals and backgrounds.

small missing energy and the soft leptons makes the transverse mass variable less efficient for suppressing the backgrounds without sacrificing the SUSY signal events.

To see the effect, we compute the transverse mass of one lepton, which is not paired with the other lepton to obtain the OSSF dilepton invariant mass, and the missing energy. See the left panel of figure 10. Since the signals are populated largely in the smaller region, we apply an upper cut instead of a lower cut on the transverse mass, $M_{\mathrm{T}}<60 \mathrm{GeV}$. Since the signal contains multiple leptons, we further attempt to use the other collider variable $M_{\mathrm{T} 2}$, which is a generalized transverse mass variable applicable in the case when there are two invisible particles in the event, for the system of an opposite-sign lepton pair and the missing energy $[145,146]$. Although it does not have any particular correlation with the sparticle masses in the signal processes, the dileptonic $t \bar{t}$ and $W W$ backgrounds exhibit the edges around $m_{W}$ as can be seen in the right panel of figure 10. By definition, the input trial mass for the invisible particle is necessary to calculate the $M_{\mathrm{T} 2}$. We set the invisible particle to be massless as it is the correct choice for the backgrounds where the neutrino is the main source of the missing energy. Among several ways of pairing the leptons, we calculate the $M_{\mathrm{T} 2}$ of all possible opposite-sign lepton pairs and choose the smallest value among them in the event. Then, an upper cut as $M_{\mathrm{T} 2}<35 \mathrm{GeV}$ is imposed similarly as $M_{\mathrm{T}}$ since the $M_{\mathrm{T} 2}$ values of the signal events are small as can be seen in the right panel of figure 10. In the CMS analysis, the $M_{\mathrm{CT}}$, so-called contransverse mass defined in ref. [147], has been also used. However, it is claimed that the $M_{\mathrm{CT}}$ is equivalent to the $M_{\mathrm{T} 2}$ in the case when the visible and invisible particles are massless $[148,149]$. Therefore, we do not apply the cut on the $M_{\mathrm{CT}}$ in our analysis.

In addition, a jet-veto cut which rejects events containing high- $p_{\mathrm{T}}$ jets can be applied. The selection cuts defined in this section seem to be already good enough for suppressing the leading-order backgrounds considered in this study. However, since we have used the 


\begin{tabular}{|l|rr|rrrrrr|}
\hline Selection cuts & BP1 & BP2 & Diboson & Triboson & $t \bar{t}$ & $t \bar{t} W / Z$ & DY & $Z+$ jet \\
\hline Basic cuts & 79.5 & 140.2 & 523.6 & 2.1 & 991.4 & 3.0 & 4966.9 & 885.6 \\
$\mathbb{E}_{\mathrm{T}}>30 \mathrm{GeV}$ & 47.2 & 96.0 & 302.2 & 1.8 & 870.7 & 2.7 & 292.7 & 123.5 \\
$\min \Delta R_{\ell+\ell^{\prime}}<0.45$ & 45.0 & 86.8 & 9.5 & 0.2 & 86.7 & 0.2 & 16.7 & 7.7 \\
$m_{\ell^{+} \ell^{\prime}}$ cuts & 30.9 & 55.1 & 3.1 & 0.04 & 25.5 & 0.03 & 0.0 & 0.0 \\
$M_{\mathrm{T}}<60 \mathrm{GeV}$ & 27.9 & 47.2 & 2.2 & 0.02 & 17.0 & 0.03 & - & - \\
$M_{\mathrm{T} 2}<35 \mathrm{GeV}$ & 24.6 & 37.3 & 1.5 & 0.01 & 6.2 & 0.02 & - & - \\
Jet-veto & 16.4 & 25.7 & 1.4 & 0.01 & 1.7 & - & - & - \\
\hline
\end{tabular}

Table 5. Number of events passed the event selection cuts defined in the text at the $20 \mathrm{fb}^{-1}$ integrated luminosity.

leading-order MC generators to simulate the SM backgrounds as well as the SUSY signals, a correct modeling of the higher-order processes like $t \bar{t}+$ jets or $W W+$ jets could affect the analysis in the real situation. The effect would not be significant for the SUSY signal events considered here since they do not have the source of jets in the matrix element level, except the initial state radiation. In such cases, the jet-veto cut, which rejects events containing high- $p_{\mathrm{T}}$ jets, would be useful to reduce the multi-jet backgrounds. In order to provide reference values for the dedicated experimental searches, we further see the effect of the jet-veto cut with the threshold $p_{\mathrm{T}}$ value of $40 \mathrm{GeV}$ on the signals of neutralinos and chargino signals on top of the other selection cuts.

To estimate the cut efficiency and the signal significance, we show the number of events that passed the cumulative event selection cuts discussed up to now in table 5 for both BP1 and BP2. When all the cuts, including jet-veto condition, are applied, the signal significance is well above the discovery criterion for both benchmark scenarios. Therefore, we anticipate that the SUSY signal of this kind of scenarios can be discovered even in the present $8 \mathrm{TeV}$ LHC data by tuning the lepton isolation parameters optimized for the non-standard lepton signals.

Finally, we show the importance of a small $\Delta R$ value in table 6 . An increase in $\Delta R$ drastically reduces the number of signal events, evidencing the need of choosing a stringent criterion for lepton isolation in order to study this scenario. Notice that this implies modification of the search strategies usually performed in ATLAS or CMS with a dedicated criterion for lepton isolation, optimised for the decay processes involving light pseudoscalar.

\section{Conclusions}

We have studied potential LHC signatures induced by the presence of very light pseudoscalar Higgs boson (in the mass range $2 m_{\tau}<m_{a_{1}^{0}}<2 m_{b}$ ) in neutralino decays in a scenario with two light scalar Higgses within the context of the NMSSM. More specifically, 


\begin{tabular}{|l|rrrrrr|}
\hline \multirow{2}{*}{ Selection cuts } & \multicolumn{3}{|c}{$\Delta R=0.1$} & \multicolumn{2}{c|}{$\Delta R=0.2$} & \multicolumn{2}{c|}{$\Delta R=0.3$} \\
& $\mathrm{BP} 1$ & $\mathrm{BP} 2$ & $\mathrm{BP} 1$ & $\mathrm{BP} 2$ & $\mathrm{BP} 1$ & $\mathrm{BP} 2$ \\
\hline Basic cuts & 79.5 & 140.2 & 36.3 & 72.5 & 17.3 & 38.8 \\
$\mathbb{E}_{\mathrm{T}}>30 \mathrm{GeV}$ & 47.2 & 96.0 & 21.5 & 48.0 & 10.2 & 25.8 \\
$\min \Delta R_{\ell^{+} \ell^{\prime}-}<0.45$ & 45.0 & 86.8 & 19.1 & 41.0 & 8.5 & 19.2 \\
$m_{\ell^{+} \ell^{\prime}-}$ cuts & 30.9 & 55.1 & 13.0 & 24.7 & 5.9 & 11.2 \\
$M_{\mathrm{T}}<60 \mathrm{GeV}$ & 27.9 & 47.2 & 12.2 & 22.0 & 5.5 & 9.7 \\
$M_{\mathrm{T} 2}<35 \mathrm{GeV}$ & 24.6 & 37.3 & 10.0 & 16.4 & 4.4 & 6.9 \\
Jet-veto & 16.4 & 25.7 & 6.4 & 11.4 & 2.9 & 5.0 \\
\hline
\end{tabular}

Table 6. Variation of the number of events that pass the selection cuts defined in the text at the $20 \mathrm{fb}^{-1}$ integrated luminosity, for various choices of the isolation criterion, $\Delta R$.

we have considered regions of the NMSSM parameter space which feature a SM-like Higgs boson in the mass range $123-127 \mathrm{GeV}$ together with another lighter one, $h_{1}^{0}$, which is mostly singlet. For the range of masses considered, the pseudoscalar predominantly decays into a pair of taus, $a_{1}^{0} \rightarrow \tau^{+} \tau^{-}$, leading to an abundance of leptons in the final state. The resulting LHC phenomenology features multi-lepton signals with missing transverse energy in the decay chains which originates from neutralino/chargino pair production, $\widetilde{\chi}_{2,3}^{0} \widetilde{\chi}_{1}^{ \pm} \rightarrow \ell^{+} \ell^{-} \ell^{ \pm}+\mathbb{E}_{\mathrm{T}}, \widetilde{\chi}_{3}^{0} \widetilde{\chi}_{1}^{ \pm} \rightarrow 2 \ell^{+} 2 \ell^{-} \ell^{ \pm}+\mathbb{E}_{\mathrm{T}}$, and $\tilde{\chi}_{i}^{0} \widetilde{\chi}_{j}^{0} \rightarrow n\left(\ell^{+} \ell^{-}\right)+\mathbb{E}_{\mathrm{T}}$, with $n=2,3,4$ for $i, j=2,3$.

We have performed a scan in the NMSSM parameter space searching for these conditions and imposing all the recent experimental constraints on the Higgs sector, sparticle masses and low-energy observables. We have further assumed that the neutralino is a component of the dark matter and imposed the observed upper bound on its relic abundance and on its elastic scattering cross section off quarks. The viable points in the parameter space feature small values of $\kappa$ and $A_{\kappa}$ and as a consequence, light singlet-like $h_{1}^{0}$ and $a_{1}^{0}$. On top of this, we have also demanded a sizable $\operatorname{BR}\left(\widetilde{\chi}_{2,3}^{0} \rightarrow \widetilde{\chi}_{1}^{0} a_{1}^{0}\right)$, which favours small values of the $\mu$ parameter since this leads to Higgsino-like neutralinos. We have distinguished between two possible scenarios, depending on whether $m_{h_{1}^{0}}<m_{h_{2}^{0}} / 2$ or $m_{h_{1}^{0}}>m_{h_{2}^{0}} / 2$, and selected two representative benchmark points.

We have then carried out a reconstruction of the signal for the selected benchmark points. The useful cuts with which the signal can be separated from the background have been determined. After imposing a set of relevant cuts together with non-standard lepton lepton separation, the resulting signal to background ratio is statistically significant at the LHC with $8 \mathrm{TeV}$ center-of-mass energy and $20 \mathrm{fb}^{-1}$ of integrated luminosity. This study suggests that the analysis of inclusive multilepton searches with missing transverse energy using the full $8 \mathrm{TeV}$ LHC data and the dedicated selection cuts can be used to explore corners of the NMSSM parameter space with multiple light Higgses. 


\section{Acknowledgments}

DGC is supported by the Ramón y Cajal program of the Spanish MICINN. CBP is supported by the CERN-Korea fellowship through the National Research Foundation of Korea. MP is supported by a MultiDark Scholarship. DGC, PG and MP thank the support of the Consolider-Ingenio 2010 programme under grant MULTIDARK CSD2009-00064, the Spanish MICINN under Grants No. FPA2009-08958 and FPA2012-34694, the Spanish MINECO "Centro de excelencia Severo Ochoa Program" under grant No. SEV-2012-0249, the Community of Madrid under Grant No. HEPHACOS S2009/ESP-1473, and the European Union under the Marie Curie-ITN Program No. PITN-GA-2009-237920.

Open Access. This article is distributed under the terms of the Creative Commons Attribution License (CC-BY 4.0), which permits any use, distribution and reproduction in any medium, provided the original author(s) and source are credited.

\section{References}

[1] U. Ellwanger, C. Hugonie and A.M. Teixeira, The next-to-minimal supersymmetric standard model, Phys. Rept. 496 (2010) 1 [arXiv:0910.1785] [inSPIRE].

[2] J.E. Kim and H.P. Nilles, The $\mu$ problem and the strong CP problem, Phys. Lett. B 138 (1984) 150 [INSPIRE].

[3] M. Drees, Supersymmetric models with extended Higgs sector, Int. J. Mod. Phys. A 4 (1989) 3635 [inSPIRE].

[4] J.R. Ellis, J.F. Gunion, H.E. Haber, L. Roszkowski and F. Zwirner, Higgs bosons in a nonminimal supersymmetric model, Phys. Rev. D 39 (1989) 844 [INSPIRE].

[5] P. Binetruy and C.A. Savoy, Higgs and top masses in a nonminimal supersymmetric theory, Phys. Lett. B 277 (1992) 453 [INSPIRE].

[6] J.R. Espinosa and M. Quirós, On Higgs boson masses in nonminimal supersymmetric standard models, Phys. Lett. B 279 (1992) 92 [INSPIRE].

[7] J.R. Espinosa and M. Quirós, Upper bounds on the lightest Higgs boson mass in general supersymmetric standard models, Phys. Lett. B 302 (1993) 51 [hep-ph/9212305] [INSPIRE].

[8] S.F. King and P.L. White, Resolving the constrained minimal and next-to-minimal supersymmetric standard models, Phys. Rev. D 52 (1995) 4183 [hep-ph/9505326] [INSPIRE].

[9] M. Bastero-Gil et al., Does LEP prefer the NMSSM?, Phys. Lett. B 489 (2000) 359 [hep-ph/0006198] [INSPIRE].

[10] R. Dermisek and J.F. Gunion, Escaping the large fine tuning and little hierarchy problems in the next to minimal supersymmetric model and $h \rightarrow$ aa decays, Phys. Rev. Lett. 95 (2005) 041801 [hep-ph/0502105] [INSPIRE].

[11] R. Dermisek and J.F. Gunion, The NMSSM solution to the fine-tuning problem, precision electroweak constraints and the largest LEP Higgs event excess, Phys. Rev. D 76 (2007) 095006 [arXiv:0705.4387] [InSPIRE].

[12] U. Ellwanger, G. Espitalier-Noel and C. Hugonie, Naturalness and fine tuning in the NMSSM: implications of early LHC results, JHEP 09 (2011) 105 [arXiv:1107.2472] [INSPIRE]. 
[13] A. Arvanitaki and G. Villadoro, A non standard model higgs at the LHC as a sign of naturalness, JHEP 02 (2012) 144 [arXiv:1112.4835] [INSPIRE].

[14] Z. Kang, J. Li and T. Li, On naturalness of the MSSM and NMSSM, JHEP 11 (2012) 024 [arXiv:1201.5305] [INSPIRE].

[15] T. Cheng et al., Toward the natural and realistic NMSSM with and without R-parity, arXiv:1207.6392 [INSPIRE].

[16] M. Perelstein and B. Shakya, XENON100 implications for naturalness in the MSSM, NMSSM and $\Lambda$-SUSY, Phys. Rev. D 88 (2013) 075003 [arXiv: 1208.0833] [InSPIRE].

[17] K. Agashe, Y. Cui and R. Franceschini, Natural islands for a $125 \mathrm{GeV}$ Higgs in the scale-invariant NMSSM, JHEP 02 (2013) 031 [arXiv:1209.2115] [INSPIRE].

[18] S. King, M. Mühlleitner, R. Nevzorov and K. Walz, Natural NMSSM Higgs bosons, Nucl. Phys. B 870 (2013) 323 [arXiv:1211.5074] [INSPIRE].

[19] ATLAS collaboration, Observation of a new particle in the search for the standard model Higgs boson with the ATLAS detector at the LHC, Phys. Lett. B 716 (2012) 1 [arXiv:1207.7214] [INSPIRE].

[20] CMS collaboration, Observation of a new boson at a mass of $125 \mathrm{GeV}$ with the CMS experiment at the LHC, Phys. Lett. B 716 (2012) 30 [arXiv:1207.7235] [INSPIRE].

[21] ATLAS collaboration, Combined measurements of the mass and signal strength of the Higgs-like boson with the ATLAS detector using up to $25 \mathrm{fb}^{-1}$ of proton-proton collision data, ATLAS-CONF-2013-014 (2013).

[22] CMS collaboration, Combination of standard model Higgs boson searches and measurements of the properties of the new boson with a mass near $125 \mathrm{GeV}$, CMS-PAS-HIG-13-005 (2013).

[23] G. Bélanger et al., Higgs bosons at 98 and $125 \mathrm{GeV}$ at LEP and the LHC, JHEP 01 (2013) 069 [arXiv:1210.1976] [InSPIRE].

[24] L. Aparicio, P. Camara, D. Cerdeno, L. Ibáñez and I. Valenzuela, The NMSSM with F-theory unified boundary conditions, JHEP 02 (2013) 084 [arXiv:1212.4808] [INSPIRE].

[25] Z. Kang, J. Li, T. Li, D. Liu and J. Shu, Probing the CP-even Higgs sector via $\mathrm{H}_{3} \rightarrow \mathrm{H}_{2} \mathrm{H}_{1}$ in the natural NMSSM, Phys. Rev. D 88 (2013) 015006 [arXiv:1301.0453] [INSPIRE].

[26] D.G. Cerdeno, P. Ghosh and C.B. Park, Probing the two light Higgs scenario in the NMSSM with a low-mass pseudoscalar, JHEP 06 (2013) 031 [arXiv:1301.1325] [INSPIRE].

[27] B. Bhattacherjee et al., Implications of $98 \mathrm{GeV}$ and $125 \mathrm{GeV}$ Higgs scenario in non-decoupling SUSY with updated ATLAS, CMS and PLANCK data, Phys. Rev. D 88 (2013) 035011 [arXiv:1305.4020] [InSPIRE].

[28] LeP Working Group for Higgs boson searches, ALEPH, DELPHi, L3, OPAL collaboration, R. Barate et al., Search for the standard model Higgs boson at LEP, Phys. Lett. B 565 (2003) 61 [hep-ex/0306033] [INSPIRE].

[29] AlEPH, DelPhi, L3, OPAL, LEP Working Group for Higgs Boson Searches collaboration, S. Schael et al., Search for neutral MSSM Higgs bosons at LEP, Eur. Phys. J. C 47 (2006) 547 [hep-ex/0602042] [INSPIRE].

[30] M. Drees, A supersymmetric explanation of the excess of Higgs-like events at the LHC and at LEP, Phys. Rev. D 86 (2012) 115018 [arXiv:1210.6507] [INSPIRE].

[31] U. Ellwanger, J.F. Gunion, C. Hugonie and S. Moretti, Towards a no lose theorem for NMSSM Higgs discovery at the LHC, hep-ph/0305109 [INSPIRE]. 
[32] U. Ellwanger, J. Gunion, C. Hugonie and S. Moretti, NMSSM Higgs discovery at the LHC, hep-ph/0401228 [INSPIRE].

[33] U. Ellwanger, J.F. Gunion and C. Hugonie, Difficult scenarios for NMSSM Higgs discovery at the LHC, JHEP 07 (2005) 041 [hep-ph/0503203] [INSPIRE].

[34] A. Djouadi et al., Benchmark scenarios for the NMSSM, JHEP 07 (2008) 002 [arXiv:0801.4321] [INSPIRE].

[35] O. Stal and G. Weiglein, Light NMSSM Higgs bosons in SUSY cascade decays at the LHC, JHEP 01 (2012) 071 [arXiv:1108.0595] [INSPIRE].

[36] F. Franke and H. Fraas, Production and decay of neutralinos in the next-to-minimal supersymmetric standard model, Z. Phys. C 72 (1996) 309 [hep-ph/9511275] [INSPIRE].

[37] S.Y. Choi, D.J. Miller and P.M. Zerwas, The neutralino sector of the next-to-minimal supersymmetric standard model, Nucl. Phys. B 711 (2005) 83 [hep-ph/0407209] [INSPIRE].

[38] K. Cheung and T.-J. Hou, Light pseudoscalar Higgs boson in neutralino decays in the next-to-minimal supersymmetric standard model, Phys. Lett. B 674 (2009) 54

[arXiv: 0809.1122] [INSPIRE].

[39] D. Das, U. Ellwanger and A.M. Teixeira, Modified signals for supersymmetry in the NMSSM with a singlino-like LSP, JHEP 04 (2012) 067 [arXiv:1202.5244] [INSPIRE].

[40] P. Nath and R.L. Arnowitt, Supersymmetric signals at the Tevatron, Mod. Phys. Lett. A 2 (1987) 331 [InSPIRE].

[41] R. Barbieri, F. Caravaglios, M. Frigeni and M.L. Mangano, Production and leptonic decays of charginos and neutralinos in hadronic collisions, Nucl. Phys. B 367 (1991) 28 [INSPIRE].

[42] J.L. Lopez, D.V. Nanopoulos, X. Wang and A. Zichichi, Supersymmetry tests at Fermilab: a proposal, Phys. Rev. D 48 (1993) 2062 [hep-ph/9211286] [INSPIRE].

[43] H. Baer, C. Kao and X. Tata, Aspects of chargino-neutralino production at the Tevatron collider, Phys. Rev. D 48 (1993) 5175 [hep-ph/9307347] [INSPIRE].

[44] J.L. Lopez, D.V. Nanopoulos, X. Wang and A. Zichichi, Supersymmetry dileptons and trileptons at the Tevatron, Phys. Rev. D 52 (1995) 142 [hep-ph/9412346] [INSPIRE].

[45] S. Mrenna, G.L. Kane, G.D. Kribs and J.D. Wells, Possible signals of constrained minimal supersymmetry at a high luminosity Fermilab Tevatron collider, Phys. Rev. D 53 (1996) 1168 [hep-ph/9505245] [INSPIRE].

[46] M. Frank and H. Saif, Trilepton signals from chargino-neutralino production at the CERN pp collider in a supersymmetric left-right model, J. Phys. G 22 (1996) 1653 [INSPIRE].

[47] H. Baer, C.-h. Chen, F. Paige and X. Tata, Supersymmetry reach of Tevatron upgrades: a comparative study, Phys. Rev. D 54 (1996) 5866 [hep-ph/9604406] [INSPIRE].

[48] D0 collaboration, B. Abbott et al., Search for the trilepton signature from the associated production of SUSY $\tilde{\chi}_{1}^{ \pm} \tilde{\chi}_{2}^{0}$ gauginos, Phys. Rev. Lett. 80 (1998) 1591 [hep-ex/9705015] [INSPIRE].

[49] V.D. Barger, C. Kao and T.-j. Li, Trilepton signal of minimal supergravity at the Tevatron including $\tau$ lepton contributions, Phys. Lett. B 433 (1998) 328 [hep-ph/9804451] [INSPIRE].

[50] V.D. Barger and C. Kao, Trilepton signature of minimal supergravity at the upgraded Tevatron, Phys. Rev. D 60 (1999) 115015 [hep-ph/9811489] [INSPIRE]. 
[51] K.T. Matchev and D.M. Pierce, Supersymmetry reach of the Tevatron via trilepton, like sign dilepton and dilepton plus $\tau$ jet signatures, Phys. Rev. D 60 (1999) 075004 [hep-ph/9904282] [INSPIRE].

[52] H. Baer, M. Drees, F. Paige, P. Quintana and X. Tata, Trilepton signal for supersymmetry at the Fermilab Tevatron revisited, Phys. Rev. D 61 (2000) 095007 [hep-ph/9906233] [INSPIRE].

[53] K.T. Matchev and D.M. Pierce, New backgrounds in trilepton, dilepton and dilepton plus $\tau$ jet SUSY signals at the Tevatron, Phys. Lett. B 467 (1999) 225 [hep-ph/9907505] [INSPIRE].

[54] M. Bisset, F. Moortgat and S. Moretti, Trilepton + top signal from chargino neutralino decays of MSSM charged Higgs bosons at the LHC, Eur. Phys. J. C 30 (2003) 419 [hep-ph/0303093] [INSPIRE].

[55] D0 collaboration, V. Abazov et al., Search for supersymmetry via associated production of charginos and neutralinos in final states with three leptons, Phys. Rev. Lett. 95 (2005) 151805 [hep-ex/0504032] [INSPIRE].

[56] CDF collaboration, T. Aaltonen et al., Search for chargino-neutralino production in p $\bar{p}$

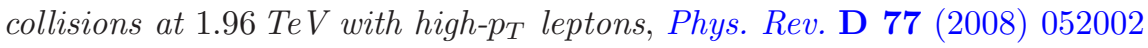
[arXiv:0711.3161] [INSPIRE].

[57] Z. Sullivan and E.L. Berger, Trilepton production at the CERN LHC: standard model sources and beyond, Phys. Rev. D 78 (2008) 034030 [arXiv:0805.3720] [INSPIRE].

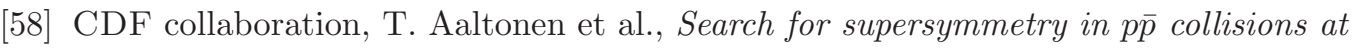
$\sqrt{s}=1.96 \mathrm{TeV}$ using the trilepton signature of chargino-neutralino production, Phys. Rev. Lett. 101 (2008) 251801 [arXiv:0808.2446] [INSPIRE].

[59] D0 collaboration, V. Abazov et al., Search for associated production of charginos and neutralinos in the trilepton final state using $2.3 \mathrm{fb}^{-1}$ of data, Phys. Lett. B 680 (2009) 34 [arXiv:0901.0646] [INSPIRE].

[60] N. Bhattacharyya and A. Datta, Tracking down the elusive charginos/neutralinos through tau leptons at the Large Hadron Collider, Phys. Rev. D 80 (2009) 055016 [arXiv:0906.1460] [INSPIRE].

[61] S. Mondal, S. Biswas, P. Ghosh and S. Roy, Exploring novel correlations in trilepton channels at the LHC for the minimal supersymmetric inverse seesaw model, JHEP 05 (2012) 134 [arXiv:1201.1556] [INSPIRE].

[62] CMS collaboration, Search for anomalous production of multilepton events in pp collisions at $\sqrt{s}=7 \mathrm{TeV}$, JHEP 06 (2012) 169 [arXiv: 1204.5341] [INSPIRE].

[63] ATLAS collaboration, Search for supersymmetry in events with three leptons and missing transverse momentum in $\sqrt{s}=7 \mathrm{TeV}$ pp collisions with the ATLAS detector, Phys. Rev. Lett. 108 (2012) 261804 [arXiv:1204.5638] [INSPIRE].

[64] ATLAS collaboration, Search for direct production of charginos and neutralinos in events with three leptons and missing transverse momentum in $\sqrt{s}=7 \mathrm{TeV}$ pp collisions with the ATLAS detector, Phys. Lett. B 718 (2013) 841 [arXiv:1208.3144] [INSPIRE].

[65] CMS collaboration, Search for electroweak production of charginos and neutralinos using leptonic final states in pp collisions at $\sqrt{s}=7$ TeV, JHEP 11 (2012) 147 [arXiv:1209.6620] [INSPIRE]. 
[66] ATLAS collaboration, Search for new phenomena in events with three charged leptons at $\sqrt{s}=7$ TeV with the ATLAS detector, Phys. Rev. D 87 (2013) 052002 [arXiv:1211.6312] [INSPIRE].

[67] CMS collaboration, Search for direct EWK production of SUSY particles in multilepton modes with 8 TeV data, CMS-PAS-SUS-12-022 (2012).

[68] ATLAS collaboration, Search for direct production of charginos and neutralinos in events with three leptons and missing transverse momentum in $13.0 \mathrm{fb}^{-1}$ of pp collisions at $\sqrt{\mathrm{s}}=8$ TeV with the ATLAS detector, ATLAS-CONF-2012-154 (2012).

[69] ATLAS collaboration, Search for direct production of charginos and neutralinos in events with three leptons and missing transverse momentum in $21 \mathrm{fb}^{-1}$ of $p p$ collisions at $\sqrt{s}=8$ TeV with the ATLAS detector, ATLAS-CONF-2013-035 (2013).

[70] CMS collaboration, A search for anomalous production of events with three or more leptons using 9.2 fb, CMS-PAS-SUS-12-026 (2012).

[71] S.P. Martin, A supersymmetry primer, in Perspectives on supersymmetry II, G.L. Kane ed. World Scientific, Singapore (2009), hep-ph/9709356 [INSPIRE].

[72] D. Cerdeno, C. Hugonie, D. Lopez-Fogliani, C. Muñoz and A. Teixeira, Theoretical predictions for the direct detection of neutralino dark matter in the NMSSM, JHEP 12 (2004) 048 [hep-ph/0408102] [INSPIRE].

[73] G. Bélanger, F. Boudjema, C. Hugonie, A. Pukhov and A. Semenov, Relic density of dark matter in the NMSSM, JCAP 09 (2005) 001 [hep-ph/0505142] [INSPIRE].

[74] J.F. Gunion, D. Hooper and B. McElrath, Light neutralino dark matter in the NMSSM, Phys. Rev. D 73 (2006) 015011 [hep-ph/0509024] [INSPIRE].

[75] D. Cerdeno, E. Gabrielli, D. Lopez-Fogliani, C. Muñoz and A. Teixeira, Phenomenological viability of neutralino dark matter in the NMSSM, JCAP 06 (2007) 008 [hep-ph/0701271] [INSPIRE].

[76] C. Hugonie, G. Bélanger and A. Pukhov, Dark matter in the constrained NMSSM, JCAP 11 (2007) 009 [arXiv:0707.0628] [INSPIRE].

[77] CoGeNT collaboration, C. Aalseth et al., Experimental constraints on a dark matter origin for the DAMA annual modulation effect, Phys. Rev. Lett. 101 (2008) 251301 [Erratum ibid. 102 (2009) 109903] [arXiv:0807.0879] [INSPIRE].

[78] D. Das and U. Ellwanger, Light dark matter in the NMSSM: upper bounds on direct detection cross sections, JHEP 09 (2010) 085 [arXiv: 1007.1151] [INSPIRE].

[79] J.-J. Cao et al., Light dark matter in NMSSM and implication on Higgs phenomenology, Phys. Lett. B 703 (2011) 292 [arXiv:1104.1754] [InSPIRE].

[80] U. Ellwanger, J.F. Gunion and C. Hugonie, NMHDECAY: a Fortran code for the Higgs masses, couplings and decay widths in the NMSSM, JHEP 02 (2005) 066 [hep-ph/0406215] [INSPIRE].

[81] U. Ellwanger and C. Hugonie, NMHDECAY 2.0: an updated program for sparticle masses, Higgs masses, couplings and decay widths in the NMSSM, Comput. Phys. Commun. 175 (2006) 290 [hep-ph/0508022] [INSPIRE].

[82] U. Ellwanger and C. Hugonie, NMSPEC: a Fortran code for the sparticle and Higgs masses in the NMSSM with GUT scale boundary conditions,

Comput. Phys. Commun. 177 (2007) 399 [hep-ph/0612134] [INSPIRE]. 
[83] F. Feroz and M. Hobson, Multimodal nested sampling: an efficient and robust alternative to MCMC methods for astronomical data analysis,

Mon. Not. Roy. Astron. Soc. 384 (2008) 449 [arXiv:0704.3704] [INSPIRE].

[84] F. Feroz, M. Hobson and M. Bridges, MultiNest: an efficient and robust Bayesian inference tool for cosmology and particle physics, Mon. Not. Roy. Astron. Soc. 398 (2009) 1601 [arXiv: 0809.3437] [INSPIRE].

[85] CMS collaboration, Search for new physics in the multijet and missing transverse momentum final state in proton-proton collisions at $\sqrt{s}=7 \mathrm{TeV}$,

Phys. Rev. Lett. 109 (2012) 171803 [arXiv:1207.1898] [InSPIRE].

[86] ATLAS collaboration, Multi-channel search for squarks and gluinos in $\sqrt{s}=7 \mathrm{TeV} p p$ collisions with the ATLAS detector, Eur. Phys. J. C 73 (2013) 2362 [arXiv:1212.6149] [INSPIRE].

[87] ATLAS SUSY Searches - 95\% CL Lower Limits.

[88] Summary of CMS SUSY results in SMS framework.

[89] Particle Data Group collaboration, J. Beringer et al., Review of particle physics, Phys. Rev. D 86 (2012) 010001 [inSPIRE].

[90] Heavy Flavor Averaging Group collaboration, D. Asner et al., Averages of b-hadron, c-hadron and $\tau$-lepton properties, arXiv:1010.1589 [INSPIRE].

[91] CMS collaboration, Search for $B_{s}^{0} \rightarrow \mu^{+} \mu^{-}$and $B^{0} \rightarrow \mu^{+} \mu^{-}$decays, JHEP 04 (2012) 033 [arXiv:1203.3976] [INSPIRE].

[92] LHCb collaboration, Strong constraints on the rare decays $B_{s} \rightarrow \mu^{+} \mu^{-}$and $B^{0} \rightarrow \mu^{+} \mu^{-}$, Phys. Rev. Lett. 108 (2012) 231801 [arXiv:1203.4493] [INSPIRE].

[93] ATLAS collaboration, Search for the decay $B_{s}^{0} \rightarrow \mu \mu$ with the ATLAS detector, Phys. Lett. B 713 (2012) 387 [arXiv:1204.0735] [INSPIRE].

[94] BABAR collaboration, J. Lees et al., Evidence of $B \rightarrow \tau \nu$ decays with hadronic $B$ tags, Phys. Rev. D 88 (2013) 031102 [arXiv: 1207.0698] [INSPIRE].

[95] Heavy Flavor Averaging Group collaboration, Y. Amhis et al., Averages of b-hadron, c-hadron and $\tau$-lepton properties as of early 2012, arXiv:1207.1158 [INSPIRE].

[96] LHCb collaboration, First evidence for the Decay $B_{s}^{0} \rightarrow \mu^{+} \mu^{-}$, Phys. Rev. Lett. 110 (2013) 021801 [arXiv:1211.2674] [INSPIRE].

[97] LHCb collaboration, Measurement of the $B_{s}^{0} \rightarrow \mu^{+} \mu^{-}$branching fraction and search for $B^{0} \rightarrow \mu^{+} \mu^{-}$decays at the LHCb experiment, Phys. Rev. Lett. 111 (2013) 101805 [arXiv:1307.5024] [INSPIRE].

[98] CMS collaboration, Measurement of the $B_{s}$ to $\mu^{+} \mu^{-}$branching fraction and search for B0 to $\mu^{+} \mu^{-}$with the CMS Experiment, Phys. Rev. Lett. 111 (2013) 101804 [arXiv:1307.5025] [INSPIRE].

[99] M. Galanti, Measurement of $B\left(B_{s}^{0} \rightarrow \mu^{+} \mu^{-}\right)$and search for $B^{0} \rightarrow \mu^{+} \mu^{-}$with $C M S$, Collider Cross Talk (2013).

[100] Muon G-2 collaboration, G. Bennett et al., Final report of the muon E821 anomalous magnetic moment measurement at BNL, Phys. Rev. D 73 (2006) 072003 [hep-ex/0602035] [INSPIRE].

[101] F. Jegerlehner and A. Nyffeler, The muon g-2, Phys. Rept. 477 (2009) 1 [arXiv:0902.3360] [INSPIRE]. 
[102] NEw (G-2) collaboration, F. Gray, Measuring the muon's anomalous magnetic moment to 0.14 ppm, J. Phys. Conf. Ser. 312 (2011) 102006 [arXiv: 1009.0799] [InSPIRE].

[103] M. Davier, A. Hoecker, B. Malaescu and Z. Zhang, Reevaluation of the hadronic contributions to the muon $g-2$ and to $\alpha_{M Z}$, Eur. Phys. J. C 71 (2011) 1515 [Erratum ibid. C 72 (2012) 1874] [arXiv: 1010.4180] [INSPIRE].

[104] K. Hagiwara, R. Liao, A.D. Martin, D. Nomura and T. Teubner, $(g-2)_{\mu}$ and $\alpha\left(M_{Z}^{2}\right)$ re-evaluated using new precise data, J. Phys. G 38 (2011) 085003 [arXiv:1105.3149] [INSPIRE].

[105] WMAP collaboration, E. Komatsu et al., Seven-year Wilkinson Microwave Anisotropy Probe (WMAP) observations: cosmological interpretation,

Astrophys. J. Suppl. 192 (2011) 18 [arXiv:1001.4538] [INSPIRE].

[106] Planck collaboration, P. Ade et al., Planck 2013 results. XVI. Cosmological parameters, arXiv: 1303.5076 [INSPIRE].

[107] CDMS-II collaboration, Z. Ahmed et al., Dark matter search results from the CDMS II experiment, Science 327 (2010) 1619 [arXiv:0912.3592] [INSPIRE].

[108] XENON10 collaboration, J. Angle et al., A search for light dark matter in XENON10 data, Phys. Rev. Lett. 107 (2011) 051301 [arXiv: 1104. 3088] [INSPIRE].

[109] CDMS, EDELWEISS collaboration, Z. Ahmed et al., Combined Limits on WIMPs from the CDMS and EDELWEISS experiments, Phys. Rev. D 84 (2011) 011102 [arXiv:1105.3377] [INSPIRE].

[110] XENON100 collaboration, E. Aprile et al., Dark matter results from 225 live days of XENON100 data, Phys. Rev. Lett. 109 (2012) 181301 [arXiv:1207.5988] [INSPIRE].

[111] CDMS collaboration, R. Agnese et al., Silicon detector dark matter results from the final exposure of CDMS II, Phys. Rev. Lett. 111 (2013) 251301 [arXiv:1304.4279] [INSPIRE].

[112] ATLAS collaboration, Measurements of the properties of the Higgs-like boson in the two photon decay channel with the ATLAS detector using $25 \mathrm{fb}^{-1}$ of proton-proton collision data, ATLAS-CONF-2013-012 (2013).

[113] ATLAS collaboration, Measurements of the properties of the Higgs-like boson in the $W W^{(*)} \rightarrow \ell \nu \ell \nu$ decay channel with the ATLAS detector using $25 \mathrm{fb}^{-1}$ of proton-proton collision data, ATLAS-CONF-2013-030 (2013).

[114] J.R. Espinosa, M. Muhlleitner, C. Grojean and M. Trott, Probing for invisible Higgs decays with global fits, JHEP 09 (2012) 126 [arXiv: 1205.6790] [INSPIRE].

[115] ATLAS collaboration, Search for invisible decays of a Higgs boson produced in association with a $Z$ boson in ATLAS, ATLAS-CONF-2013-011 (2013).

[116] CMS collaboration, Search for an invisible Higgs boson, CMS-PAS-HIG-13-013 (2013).

[117] CMS collaboration, Search for invisible Higgs produced in association with a $Z$ boson, CMS-PAS-HIG-13-018 (2013).

[118] G. Bélanger, B. Dumont, U. Ellwanger, J. Gunion and S. Kraml, Status of invisible Higgs decays, Phys. Lett. B 723 (2013) 340 [arXiv:1302.5694] [InSPIRE].

[119] J. Ellis and T. You, Updated global analysis of Higgs couplings, JHEP 06 (2013) 103 [arXiv:1303.3879] [INSPIRE].

[120] G. Bélanger, B. Dumont, U. Ellwanger, J. Gunion and S. Kraml, Global fit to Higgs signal strengths and couplings and implications for extended Higgs sectors,

Phys. Rev. D 88 (2013) 075008 [arXiv:1306.2941] [INSPIRE]. 
[121] CMS collaboration, Search for the Higgs boson decaying to invisible particles produced in association with $Z$ bosons decaying to bottom quarks, CMS-PAS-HIG-13-028 (2013).

[122] M. Ciuchini, G. Degrassi, P. Gambino and G. Giudice, Next-to-leading QCD corrections to $B \rightarrow X_{s} \gamma$ in supersymmetry, Nucl. Phys. B 534 (1998) 3 [hep-ph/9806308] [INSPIRE].

[123] G. D'Ambrosio, G. Giudice, G. Isidori and A. Strumia, Minimal flavor violation: an effective field theory approach, Nucl. Phys. B 645 (2002) 155 [hep-ph/0207036] [InSPIRE].

[124] M. Misiak et al., Estimate of $B(\bar{B} \rightarrow X(s) \gamma)$ at $O\left(\alpha_{s}^{2}\right)$, Phys. Rev. Lett. 98 (2007) 022002 [hep-ph/0609232] [INSPIRE].

[125] M. Misiak and M. Steinhauser, NNLO QCD corrections to the $\bar{B} \rightarrow X_{s} \gamma$ matrix elements using interpolation in $m_{c}$, Nucl. Phys. B 764 (2007) 62 [hep-ph/0609241] [INSPIRE].

[126] M. Endo, K. Hamaguchi, S. Iwamoto, K. Nakayama and N. Yokozaki, Higgs mass and muon anomalous magnetic moment in the $\mathrm{U}(1)$ extended MSSM, Phys. Rev. D 85 (2012) 095006 [arXiv:1112.6412] [InSPIRE].

[127] M. Endo, K. Hamaguchi, S. Iwamoto and T. Yoshinaga, Muon $g-2$ vs. LHC in supersymmetric models, JHEP 01 (2014) 123 [arXiv:1303.4256] [INSPIRE].

[128] B.A. Dobrescu and K.T. Matchev, Light axion within the next-to-minimal supersymmetric standard model, JHEP 09 (2000) 031 [hep-ph/0008192] [INSPIRE].

[129] M. Bahr et al., HERWIG++ physics and manual, Eur. Phys. J. C 58 (2008) 639 [arXiv:0803.0883] [INSPIRE].

[130] K. Arnold et al., HERWIG++ 2.6 release note, arXiv: 1205.4902 [INSPIRE].

[131] J. Pumplin et al., New generation of parton distributions with uncertainties from global QCD analysis, JHEP 07 (2002) 012 [hep-ph/0201195] [INSPIRE].

[132] J. de Favereau et al., DELPHES 3, a modular framework for fast simulation of a generic collider experiment, arXiv:1307.6346 [INSPIRE].

[133] M. Cacciari, G.P. Salam and G. Soyez, The anti- $k_{t}$ jet clustering algorithm, JHEP 04 (2008) 063 [arXiv:0802.1189] [INSPIRE].

[134] ATLAS collaboration, Search for direct production of charginos and neutralinos in events with three leptons and missing transverse momentum in $21 \mathrm{fb}^{-1}$ of pp collisions at $\sqrt{s}=8 \mathrm{TeV}$ with the ATLAS detector, ATLAS-CONF-2013-035 (2013).

[135] CMS collaboration, Search for direct EWK production of SUSY particles in multilepton modes with 8TeV data, CMS-PAS-SUS-12-022 (2012).

[136] J. Alwall, M. Herquet, F. Maltoni, O. Mattelaer and T. Stelzer, MadGraph 5: going beyond, JHEP 06 (2011) 128 [arXiv:1106.0522] [INSPIRE].

[137] CMS collaboration, Measurement of the $W W, W Z$ and $Z Z$ cross sections at CMS, CMS-PAS-EWK-11-010 (2011).

[138] CMS collaboration, Measurement of $W^{+} W^{-}$and $Z Z$ production cross sections in $p p$ collisions at $\sqrt{s}=8 \mathrm{TeV}$, Phys. Lett. B 721 (2013) 190 [arXiv:1301.4698] [INSPIRE].

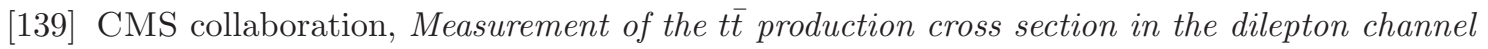
in pp collisions at $\sqrt{s}=7 \mathrm{TeV}$, JHEP 11 (2012) 067 [arXiv:1208.2671] [INSPIRE].

[140] D. Lopez-Fogliani and C. Muñoz, Proposal for a new minimal supersymmetric standard model, Phys. Rev. Lett. 97 (2006) 041801 [hep-ph/0508297] [INSPIRE].

[141] N. Escudero, D.E. Lopez-Fogliani, C. Muñoz and R.R. de Austri, Analysis of the parameter space and spectrum of the mu nu SSM, JHEP 12 (2008) 099 [arXiv:0810.1507] [INSPIRE]. 
[142] J. Fidalgo, D.E. Lopez-Fogliani, C. Muñoz and R.R. de Austri, The Higgs sector of the $\mu \nu$ SSM and collider physics, JHEP 10 (2011) 020 [arXiv:1107.4614] [INSPIRE].

[143] P. Ghosh et al., Probing the $\mu$ from $\nu$ supersymmetric standard model with displaced multileptons from the decay of a Higgs boson at the LHC, Phys. Rev. D 88 (2013) 015009 [arXiv:1211.3177] [INSPIRE].

[144] B.C. Allanach, SOFTSUSY: a program for calculating supersymmetric spectra, Comput. Phys. Commun. 143 (2002) 305 [hep-ph/0104145] [INSPIRE].

[145] C. Lester and D. Summers, Measuring masses of semiinvisibly decaying particles pair produced at hadron colliders, Phys. Lett. B 463 (1999) 99 [hep-ph/9906349] [INSPIRE].

[146] A. Barr, C. Lester and P. Stephens, $m(T 2)$ : the truth behind the glamour, J. Phys. G 29 (2003) 2343 [hep-ph/0304226] [INSPIRE].

[147] D.R. Tovey, On measuring the masses of pair-produced semi-invisibly decaying particles at hadron colliders, JHEP 04 (2008) 034 [arXiv:0802.2879] [INSPIRE].

[148] C.G. Lester, The stransverse mass, m(T2), in special cases, JHEP 05 (2011) 076 [arXiv:1103.5682] [INSPIRE].

[149] C.H. Lally and C.G. Lester, Properties of $m(T 2)$ in the massless limit, arXiv:1211.1542 [INSPIRE]. 